Pacific Northwest

National Laboratory

Operated by Battelle for the

U.S. Department of Energy

\title{
A Compendium of Transfer Factors for Agricultural and Animal Products
}
L. H. Staven
K. Rhoads
B. A. Napier
D. L. Strenge

June 2003

Prepared for the U.S. Department of Energy under Contract DE-AC06-76RL01830 


\title{
DISCLAIMER
}

This report was prepared as an account of work sponsored by an agency of the United States Government. Neither the United States Government nor any agency thereof, nor Battelle Memorial Institute, nor any of their employees, makes any warranty, express or implied, or assumes any legal liability or responsibility for the accuracy, completeness, or usefulness of any information, apparatus, product, or process disclosed, or represents that its use would not infringe privately owned rights. Reference herein to any specific commercial product, process, or service by trade name, trademark, manufacturer, or otherwise does not necessarily constitute or imply its endorsement, recommendation, or favoring by the United States Government or any agency thereof, or Battelle Memorial Institute. The views and opinions of authors expressed herein do not necessarily state or reflect those of the United States Government or any agency thereof.

\author{
PACIFIC NORTHWEST NATIONAL LABORATORY \\ operated by \\ BATTELLE \\ for the \\ UNITED STATES DEPARTMENT OF ENERGY \\ under Contract DE-AC06-76RL01830
}

Printed in the United States of America

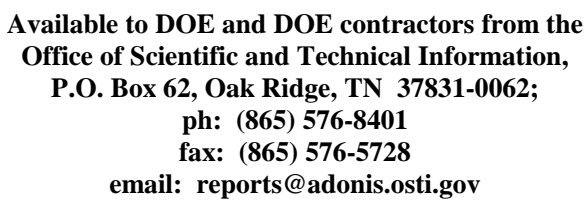

Available to the public from the National Technical Information Service, U.S. Department of Commerce, 5285 Port Royal Rd., Springfield, VA 22161 ph: (800) 553-6847 fax: $(703) 605-6900$

email: orders@ntis.fedworld.gov

online ordering: http://www.ntis.gov/ordering.htm 


\section{Summary}

Transfer factors are used in radiological risk assessments to estimate the amount of radioactivity that could be present in a food crop or organism based on the calculated concentration in the source medium (i.e., soil or animal feed). By calculating the concentration in the food, the total intake can be estimated and a dose calculated as a result of the annual intake. This report compiles transfer factors for radiological risk assessments, using common food products, including meats, eggs, and plants. Transfer factors used are most often selected from recommended values listed by national or international organizations for use in radiological food chain transport calculations. Several methods of estimation and extrapolation are used for radionuclides not listed in the primary information sources. Tables of transfer factors are listed by element and information source for leafy vegetables, fruit, root vegetables, grain, beef, poultry, milk, eggs, fresh and saltwater fish as well as crustaceans, mollusks, and aquatic plants. 


\section{Acknowledgements}

The authors are indebted to Joe Soldat and Dave Baker without whose work in pathway analysis, this compendium would not be possible. This program was funded through the Surface Environmental Surveillance Program at Hanford and the Environmental Protection Agency, Office of Radiation and Indoor Air.

This document was developed in conjunction with the upgrade to the GENII system of computer codes. The GENII computer code was developed to incorporate the internal dosimetry models recommended by the International Commission on Radiological Protection (ICRP) and the radiological risk estimating procedures of Federal Guidance Report 13 into updated versions of existing environmental pathway analysis models. GENII and the Windows ${ }^{\mathrm{TM}}$ compatible GENII-Version 2 provide state-of-theart, technically peer-reviewed, documented set of programs for calculating radiation dose and risk from radionuclides released to the environment. Upgrading the codes to GENII-Version 2 provided the opportunity to update and further document the library of transfer factors that support the environmental pathway analyses. 


\section{Contents}

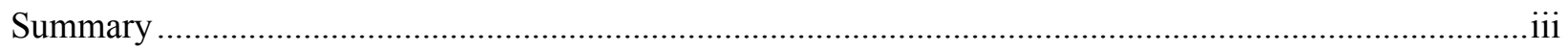

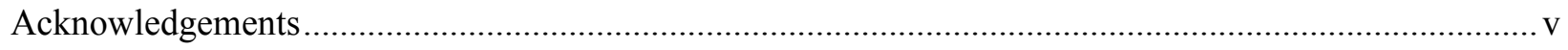

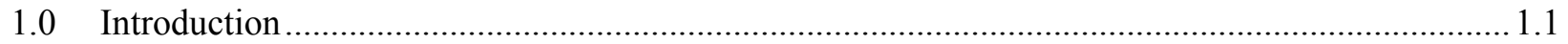

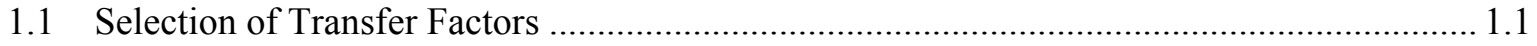

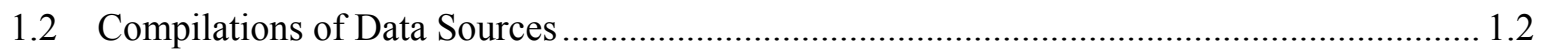

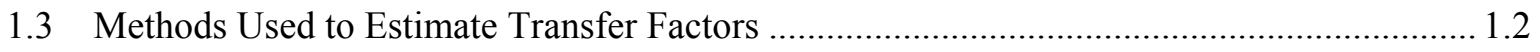

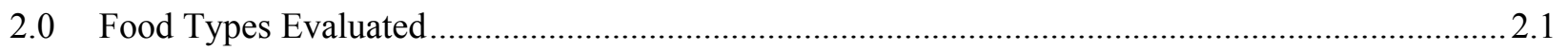

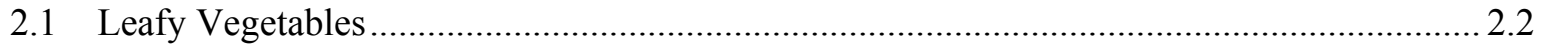

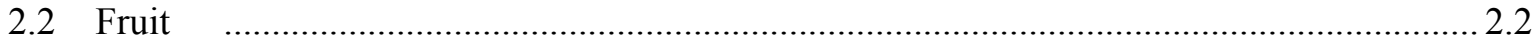

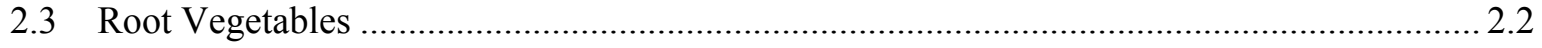

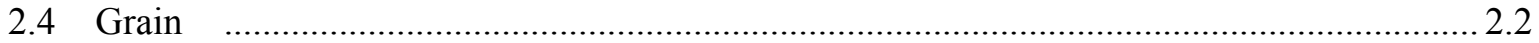

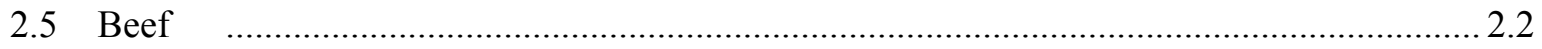

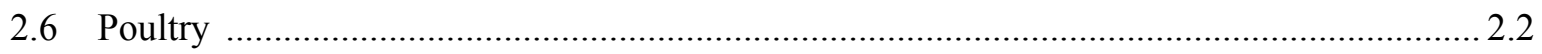

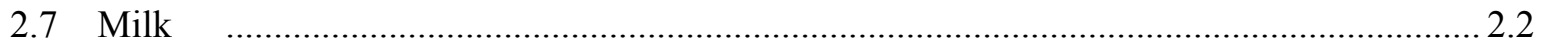

2.8 Egg

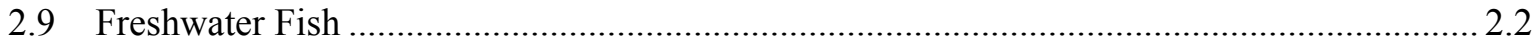

2.10 Saltwater Fish, Crustaceans, Molluscs, and Water Plants ................................................. 2.2

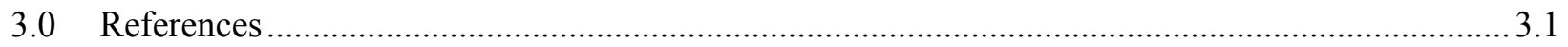

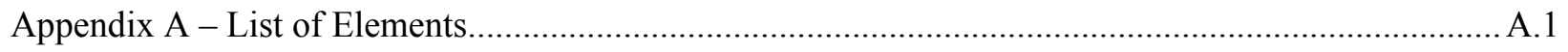




\section{Figure}

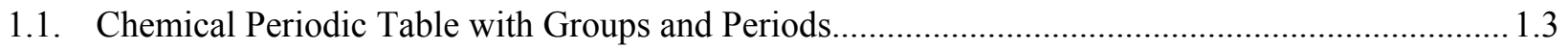

\section{Tables}

2.1. Dry-to-Wet Weight Conversion Factors for Food Products

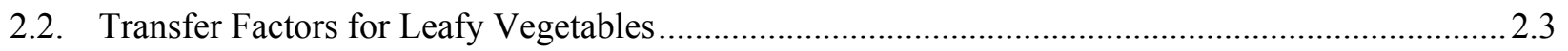

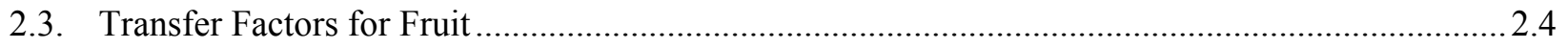

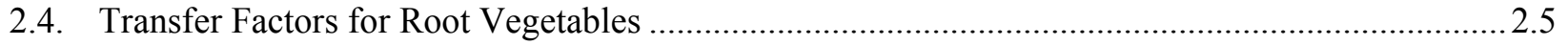

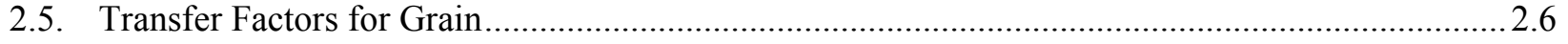

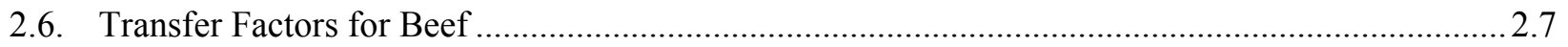

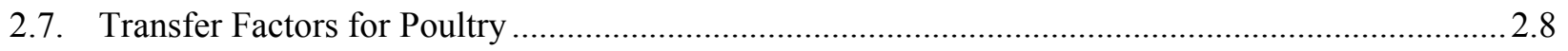

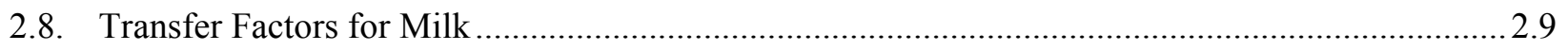

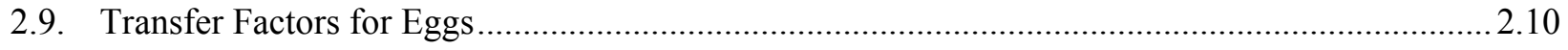

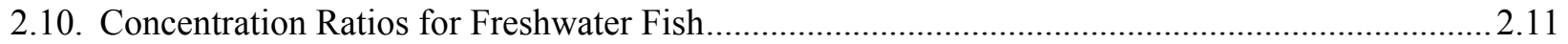

2.11. Concentration Ratios for Saltwater Fish, as Compiled in GENII .............................................2.12

2.12. Concentration Ratios for Saltwater and Freshwater Crustaceans,

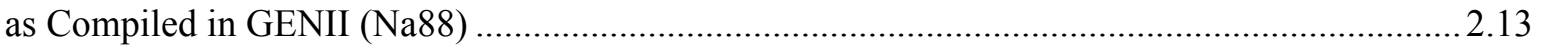

2.13. Concentration Ratios for Molluscs, as Compiled in GENII ( $\mathrm{Na} 88)$..........................................2.14

2.14. Concentration Ratios for Water Plants, as Compiled in GENII (Na88)...................................2.15 


\subsection{Introduction}

Transfer factors, also known as concentration ratios or bioaccumulation factors, are the ratio of the concentration of an element in an organism of interest, such as plants and food products, to the concentration in the source medium, such as soil (for plants), plant forage (for animals), or water (for fish, crustaceans, mollusks, and aquatic plants). Transfer factors are used in calculating radionuclide uptake by animals and humans via food pathways. Measurements show that similar concentrations of various radionuclides in soil or water do not produce the same concentrations once they are taken up into tissue. Transfer factors are used in radiological risk assessments to estimate the amount of radioactivity that could be present in a food crop or organism based on the calculated concentration in the source medium (i.e., soil or animal feed). By calculating the concentration in the food, the total intake can be estimated and a dose calculated as a result of the annual intake. This report compiles transfer factors for radiological risk assessments using common food products, including meats, eggs, and plants.

The transfer factor applies to long-term, chronic exposure of plants and animals and is ideally measured at equilibrium. For plant products, transfer factors relate the concentration of an element in soil to the element's concentration in the plant; for animals, the transfer factor relates concentration in animal feed to animal products; and for fish, the transfer factor relates the concentration in water to the concentration in fish muscle tissue. In terms of radionuclides, the transfer factor is used to calculate how many becquerel per kilogram (Bq per $\mathrm{kg}$ ) of soil are transferred to the edible dry plant product. For vegetables, the units are $\mathrm{kg}$ soil $/ \mathrm{kg}$ edible dry plants. For animal products, the transfer factor relates the daily intake to that of the animal product. Units for milk are $\mathrm{Bq} / \mathrm{L}$ per $\mathrm{Bq}$ intake/d. For animal and fish meat products, transfer factors are measured in wet weight animal product: $\mathrm{Bq} / \mathrm{kg}$ muscle per $\mathrm{Bq} / \mathrm{d}$ intake for meat and $\mathrm{Bq} / \mathrm{kg}$ muscle per $\mathrm{Bq} / \mathrm{L}$ for fish. For water plants, the units are expressed in wet weight $\mathrm{Bq} / \mathrm{kg}$ plant per $\mathrm{Bq} / \mathrm{L}$.

Numerous studies have been undertaken to quantify transfer factors for specific chemical elements as a function of food type. These studies have been compiled in several publications (see "Compilations of Data Sources," below).

\subsection{Selection of Transfer Factors}

Transfer factors compiled for this report are generally selected from recommended values listed by national or international organizations for use in radiological food chain transport calculations. (In some cases, where data are available, very recent references for specific values are preferred to compiled recommendations.) In general, the recommended values from more recent compilations are selected rather than those in older publications. The primary data sources are also cited where identified in the compilation document. In many cases, where neither a primary source of data nor a recommended value in a compilation is available, transfer factors for such elements are extrapolated from experimental data based on chemical similarities. 


\subsection{Compilations of Data Sources}

A hierarchy of data sources was established to select recommended values for transfer factors. The most recent and comprehensive references are given priority. The first reference chosen is the International Atomic Energy Agency's (IAEA) Technical Report Series \#364, Handbook of Parameter Values for the Prediction of Radionuclide Transfer in Temperate Environments (IAEA 1994). This document encompasses a wide variety of plant types and is the result of extensive background investigations. It is based on data compiled by the International Union of Radioecologists. The second reference given consideration is NUREG/CR-5512, Residual Radioactive Contamination from Decommissioning: Technical Basis for Translating Contamination Levels to Annual Total Effective Dose Equivalent (Kennedy and Strenge 1992) because of its large set of data and traceable references. Three other references are also used to fill in data: NCRP Report \#123 (1996), Screening Models for Releases of Radionuclides to Atmosphere, Surface Water, and Ground; the library from the GENII system of computer codes (Napier et al. 1988); and the series of documents by Coughtrey and Thorne, Radionuclide Distribution and Transport in Terrestrial and Aquatic Ecosystems, Vols. 1-6 (1983). NCRP Report \#123 is chosen because it is a generally accepted reference for a generic model. The GENII values have been in use for a decade to model the semi-arid environment at the Hanford Site and are reasonably well documented. Although the methodology for the Coughtrey and Thorne values is somewhat different, resulting in high values for transfer factors, it is necessary to use their values to fill in data where no other information is available. When no referenceable documents are available, data are derived based on chemical groupings in the periodic table of the elements as described below.

\subsection{Methods Used to Estimate Transfer Factors}

Experimental data are not available for all elements for all food types. Therefore, several methods are employed to estimate transfer factors for elements and food types where data are lacking. In some cases, parameters from one vegetable type are applied to remaining vegetable types for the same element. In these cases, no adjustments are made for the wet/dry ratios for specific food types, due to the relatively large uncertainty inherent in applying the transfer factor from one food type to another. In other cases, chemical similarities among elements are the basis for estimating the transfer factor. If two or more transfer factors are available for elements in a chemical group, the geometric mean is calculated from the chemical group for the given food type (Figure 1.1). Where data are available for only one element in a chemical group, the transfer factor for that element is applied to other elements in the same chemical group. Specific cases are listed in the section below. For the actinides, many of the values from americium are applied to other elements for which parameters are not available. Cerium is the surrogate element for other lanthanides for which values are not known. 


\begin{tabular}{|c|c|c|c|c|c|c|c|c|c|c|c|c|c|c|c|c|c|c|}
\hline & IA & & & & & & & & & & & & & & & & & $\begin{array}{l}\text { Noble } \\
\text { Gases }\end{array}$ \\
\hline & $\begin{array}{l}1 \\
\mathrm{H} \\
\end{array}$ & IIA & & & & & & & & & & & IIIA & IVA & VA & VIA & VIIA & $\begin{array}{c}2 \\
\mathrm{He} \\
\end{array}$ \\
\hline & $\begin{array}{c}3 \\
\mathrm{Li} \\
\end{array}$ & $\begin{array}{c}4 \\
\mathrm{Be}\end{array}$ & & & & & & & & & & & $\begin{array}{l}5 \\
\mathrm{~B} \\
\end{array}$ & $\begin{array}{l}6 \\
\mathrm{C} \\
\end{array}$ & $\begin{array}{c}7 \\
\mathrm{~N}\end{array}$ & $\begin{array}{l}8 \\
\mathrm{O}\end{array}$ & $\begin{array}{l}9 \\
F\end{array}$ & $\begin{array}{l}10 \\
\mathrm{Ne}\end{array}$ \\
\hline Period & $\begin{array}{c}11 \\
\mathrm{Na} \\
\end{array}$ & $\begin{array}{c}12 \\
\mathrm{Mg}\end{array}$ & III & |- & & & & inds & & & & & $\begin{array}{l}13 \\
\mathrm{Al} \\
\end{array}$ & $\begin{array}{l}14 \\
\mathrm{Si} \\
\end{array}$ & $\begin{array}{l}15 \\
\mathrm{P} \\
\end{array}$ & $\begin{array}{c}16 \\
\mathrm{~S} \\
\end{array}$ & $\begin{array}{l}17 \\
\mathrm{Cl} \\
\end{array}$ & $\begin{array}{l}18 \\
\mathrm{Ar}\end{array}$ \\
\hline IV & $\begin{array}{l}19 \\
\mathrm{~K} \\
\end{array}$ & $\begin{array}{l}20 \\
\mathrm{Ca} \\
\end{array}$ & $\begin{array}{l}21 \\
\mathrm{Sc} \\
\end{array}$ & $\begin{array}{r}22 \\
\mathrm{Ti} \\
\end{array}$ & $\begin{array}{l}23 \\
\mathrm{~V}\end{array}$ & $\begin{array}{l}24 \\
\mathrm{Cr} \\
\end{array}$ & $\begin{array}{r}25 \\
\mathrm{Mn} \\
\end{array}$ & $\begin{array}{l}26 \\
\mathrm{Fe} \\
\end{array}$ & $\begin{array}{l}27 \\
\mathrm{Co} \\
\end{array}$ & $\begin{array}{l}28 \\
\mathrm{Ni} \\
\end{array}$ & $\begin{array}{l}29 \\
\mathrm{Cu} \\
\end{array}$ & $\begin{array}{l}30 \\
\mathrm{Zn} \\
\end{array}$ & $\begin{array}{l}31 \\
\mathrm{Ga} \\
\end{array}$ & $\begin{array}{l}32 \\
\mathrm{Ge} \\
\end{array}$ & $\begin{array}{l}33 \\
\text { As } \\
\end{array}$ & $\begin{array}{l}34 \\
\mathrm{Se} \\
\end{array}$ & $\begin{array}{l}35 \\
\mathrm{Br}\end{array}$ & $\begin{array}{l}36 \\
\mathrm{Kr} \\
\end{array}$ \\
\hline V & $\begin{array}{l}37 \\
\mathrm{Rb} \\
\end{array}$ & $\begin{array}{l}38 \\
\mathrm{Sr} \\
\end{array}$ & $\begin{array}{c}39 \\
\mathrm{Y} \\
\end{array}$ & $\begin{array}{l}40 \\
\mathrm{Zr}\end{array}$ & $\begin{array}{l}41 \\
\mathrm{Nb}\end{array}$ & $\begin{array}{c}42 \\
\text { Mo } \\
\end{array}$ & $\begin{array}{l}43 \\
\mathrm{Tc} \\
\end{array}$ & $\begin{array}{l}44 \\
\mathrm{Ru} \\
\end{array}$ & $\begin{array}{l}45 \\
\mathrm{Rh} \\
\end{array}$ & $\begin{array}{l}46 \\
\mathrm{Pd} \\
\end{array}$ & $\begin{array}{r}47 \\
\mathrm{Ag} \\
\end{array}$ & $\begin{array}{l}48 \\
\mathrm{Cd} \\
\end{array}$ & $\begin{array}{l}49 \\
\text { In }\end{array}$ & $\begin{array}{l}50 \\
\text { Sn } \\
\end{array}$ & $\begin{array}{l}51 \\
\mathrm{Sb} \\
\end{array}$ & $\begin{array}{l}52 \\
\mathrm{Te} \\
\end{array}$ & $\begin{array}{c}53 \\
\mathrm{I}\end{array}$ & $\begin{array}{l}54 \\
\mathrm{Xe} \\
\end{array}$ \\
\hline VI & $\begin{array}{l}55 \\
\mathrm{Cs} \\
\end{array}$ & $\begin{array}{l}56 \\
\mathrm{Ba} \\
\end{array}$ & $\begin{array}{l}57 \\
\mathrm{La} \\
\end{array}$ & $\begin{array}{l}72 \\
\mathrm{Hf}\end{array}$ & $\begin{array}{l}73 \\
\mathrm{Ta} \\
\end{array}$ & $\begin{array}{l}74 \\
\text { W }\end{array}$ & $\begin{array}{l}75 \\
\mathrm{Re} \\
\end{array}$ & $\begin{array}{l}76 \\
\text { Os } \\
\end{array}$ & $\begin{array}{l}77 \\
\text { Ir } \\
\end{array}$ & $\begin{array}{l}78 \\
\mathrm{Pt} \\
\end{array}$ & $\begin{array}{r}79 \\
\mathrm{Au} \\
\end{array}$ & $\begin{array}{r}80 \\
\mathrm{Hg} \\
\end{array}$ & $\begin{array}{l}81 \\
\mathrm{Tl} \\
\end{array}$ & $\begin{array}{l}82 \\
\mathrm{~Pb} \\
\end{array}$ & $\begin{array}{l}83 \\
\mathrm{Bi} \\
\end{array}$ & $\begin{array}{l}84 \\
\text { Po } \\
\end{array}$ & $\begin{array}{l}85 \\
\text { At }\end{array}$ & $\begin{array}{l}86 \\
\mathrm{Rn} \\
\end{array}$ \\
\hline VII & $\begin{array}{l}87 \\
\mathrm{Fr} \\
\end{array}$ & $\begin{array}{l}88 \\
\mathrm{Ra} \\
\end{array}$ & $\begin{array}{l}89 \\
\mathrm{Ac}\end{array}$ & $\begin{array}{c}104 \\
\mathrm{Rf} \\
\end{array}$ & $\begin{array}{l}105 \\
\mathrm{Ha} \\
\end{array}$ & $\begin{array}{l}106 \\
\mathrm{Sg}\end{array}$ & $\begin{array}{l}107 \\
\text { Ns }\end{array}$ & $\begin{array}{c}108 \\
\mathrm{Hs}\end{array}$ & $\begin{array}{l}109 \\
\mathrm{Mt}\end{array}$ & & & & & & & & & \\
\hline
\end{tabular}

\begin{tabular}{|c|c|c|c|c|c|c|c|c|c|c|c|c|c|c|}
\hline Lanthanides & $\begin{array}{l}58 \\
\mathrm{Ce} \\
\end{array}$ & $\begin{array}{l}59 \\
\operatorname{Pr}\end{array}$ & $\begin{array}{r}60 \\
\mathrm{Nd}\end{array}$ & $\begin{array}{c}61 \\
\mathrm{Pm}\end{array}$ & $\begin{array}{r}62 \\
\mathrm{Sm} \\
\end{array}$ & $\begin{array}{l}63 \\
\mathrm{Eu}\end{array}$ & $\begin{array}{c}64 \\
\mathrm{Gd}\end{array}$ & $\begin{array}{l}65 \\
\mathrm{~Tb}\end{array}$ & $\begin{array}{l}66 \\
\text { Dy }\end{array}$ & $\begin{array}{l}67 \\
\text { Ho } \\
\end{array}$ & $\begin{array}{l}68 \\
\mathrm{Er}\end{array}$ & $\begin{array}{r}69 \\
\text { Tm } \\
\end{array}$ & $\begin{array}{l}70 \\
\mathrm{Yb}\end{array}$ & $\begin{array}{l}71 \\
\mathrm{Lu}\end{array}$ \\
\hline tinides & $\begin{array}{l}90 \\
\text { Th }\end{array}$ & $\begin{array}{l}91 \\
\mathrm{~Pa}\end{array}$ & $\begin{array}{c}92 \\
\mathrm{U}\end{array}$ & $\begin{array}{c}93 \\
\mathrm{~Np}\end{array}$ & $\begin{array}{l}94 \\
\mathrm{Pu}\end{array}$ & $\begin{array}{c}95 \\
\text { Am }\end{array}$ & $\begin{array}{c}96 \\
\mathrm{Cm}\end{array}$ & $\begin{array}{l}97 \\
\mathrm{Bk}\end{array}$ & $\begin{array}{l}98 \\
\mathrm{Cf}\end{array}$ & $\begin{array}{l}99 \\
\text { Es }\end{array}$ & $\begin{array}{l}100 \\
\text { Fm }\end{array}$ & $\begin{array}{l}101 \\
\mathrm{Md}\end{array}$ & $\begin{array}{c}102 \\
\text { No }\end{array}$ & $\begin{array}{c}103 \\
\mathrm{Lr}\end{array}$ \\
\hline
\end{tabular}

Figure 1.1. Chemical Periodic Table with Groups and Periods. A listing of the chemicals and their symbols is in Appendix A. 


\subsection{Food Types Evaluated}

For this compendium of data, foods are grouped into the following types: beef, milk, poultry, eggs, fish, leafy vegetables, other (root) vegetables, fruit, and grain. Also included are transfer factors from the GENII code for saltwater fish, crustaceans, molluscs and water plants. When more than one food is listed for a given food type (e.g., spinach, cabbage, and lettuce are all listed as "leafy vegetables"), the transfer factor for each food is weighted by the U.S. Department of Agriculture annual per capita consumption rate (USDA 1983), and a weighted average of transfer factors is reported for that food type.

Plant transfer factors from the IAEA Handbook \#364, NUREG/CR-5512, GENII, and Coughtrey and Thorne are based on dry weight data (Bq per $\mathrm{kg}$ dry crop edible product) $/(\mathrm{Bq} / \mathrm{kg}$ dry soil in the upper $20 \mathrm{~cm}$ ). When data are presented on a wet weight basis, they are converted to dry weight using conversion factors found in Table 2.1. A wilted plant from moist soil is likely to produce a much different transfer factor than a freshly harvested plant from drier soil. By analyzing dry weight plant-tosoil transfer factors only, the variability due to moisture content of plants and soil is eliminated.

Table 2.1. Dry-to-Wet Weight Conversion Factors for Food Products ${ }^{(a)}$

\begin{tabular}{|c|c|}
\hline Plant Type & Conversion Factor \\
\hline Leafy vegetables & 0.2 \\
\hline Other vegetables & 0.25 \\
\hline Other/root vegetables & 0.25 \\
\hline Fruit & 0.18 \\
\hline Grain & 0.91 \\
\hline \multicolumn{2}{|l|}{ Beef } \\
\hline Forage & 0.22 \\
\hline Stored hay & 0.22 \\
\hline Stored grain & 0.91 \\
\hline \multicolumn{2}{|l|}{ Poultry } \\
\hline Forage & 0.22 \\
\hline Stored hay & 0.22 \\
\hline Stored grain & 0.91 \\
\hline \multicolumn{2}{|l|}{ Dairy } \\
\hline Forage & 0.22 \\
\hline Stored hay & 0.22 \\
\hline Stored grain & 0.91 \\
\hline \multicolumn{2}{|l|}{ Eggs } \\
\hline Forage & 0.22 \\
\hline Stored hay & 0.22 \\
\hline Stored grain & 0.91 \\
\hline \multicolumn{2}{|c|}{$\begin{array}{l}\text { (a) From Kennedy and Strenge (1992), as adapted from Till and } \\
\text { Meyer (1983) Table 5-16. Factors in Till \& Meyer are expressed as } \\
\text { fresh (wet) to dry and were expressed by Kennedy and Strenge (1992) } \\
\text { and in this report, as inversions for the purposes of converting from wet } \\
\text { to dry weight. }\end{array}$} \\
\hline
\end{tabular}


All terrestrial plant transfer factors presented in this report are based on dry weight. Animal products and aquatic plants are presented on a wet weight or volume (milk) basis.

Tables 2.2 to 2.14 list a) the recommended transfer factors by food or crop type, b) the compilation from which the value is taken, and c) the original sources of data as cited in each compilation document. Some transfer factors, described below, are estimated for special cases without direct primary references.

\subsection{Leafy Vegetables}

The soil-to-leafy-vegetables transfer factors for N, Hf, Ta, and Au are calculated as the geometric means of the transfer factors in their respective periodic table groups. All the lanthanide elements are assigned the value for $\mathrm{Ce}$. Actinium (Ac), $\mathrm{Pa}$, and $\mathrm{Cf}$ are assigned the leafy vegetables transfer factor from Am.

\subsection{Fruit}

The soil-to-fruit transfer factors for $\mathrm{N}, \mathrm{Os}, \mathrm{Au}$, and $\mathrm{Hg}$ are calculated based on the geometric mean of the transfer factors in their respective groups on the periodic table. Hafnium (Hf) and Ta are assigned values equal to those for $\mathrm{Zr}$ and $\mathrm{Nb}$, respectively. All the lanthanide elements are assigned the value for $\mathrm{Ce}$.

\subsection{Root Vegetables}

The soil-to-root transfer factors for $\mathrm{N}$ and $\mathrm{Au}$ are calculated as the geometric mean of the transfer factors in their respective periodic table groups. Hafnium (Hf) and Ta are based on a single established value in their respective chemical groups. The root transfer factors for $\mathrm{Ac}, \mathrm{Pa}$, and $\mathrm{Cf}$ are based on $\mathrm{Am}$. All lanthanide elements are assigned the value for Ce.

\subsection{Grain}

The soil-to-grain transfer factors for $\mathrm{Be}, \mathrm{N}$, and $\mathrm{Hg}$ are calculated as the geometric mean of the transfer factors in their respective periodic table groups. Silver (Ag), Hf, Ta, and Au transfer factors for grain are based on the single known value in their respective chemical groups. All the lanthanide elements are assigned the value for $\mathrm{Ce}$. The grain transfer factors for $\mathrm{Ac}, \mathrm{Pa}$, and $\mathrm{Cf}$ are based on $\mathrm{Am}$.

\subsection{Beef}

The Ta transfer factor for feed-to-beef is set equal to the $\mathrm{Nb}$ value, and all lanthanides are based on Ce. Unknown actinides, $\mathrm{Ac}, \mathrm{Pa}$, and $\mathrm{Cf}$ values are set equal to Am values for their respective food types. All other values are based on the recommended values in a compilation document. 
Table 2.2. Transfer Factors for Leafy Vegetables (Bq/kg leafy vegetables per Bq/ $/ \mathrm{kg}$ soil)

\begin{tabular}{|c|c|c|c|c|c|c|c|}
\hline $\begin{array}{c}\mathrm{Z} \text { and } \\
\text { Element }\end{array}$ & $\begin{array}{c}\text { Recommended } \\
\text { Value (Dry } \\
\text { Weight) }\end{array}$ & $\begin{array}{c}\text { Compiled } \\
\text { in }\end{array}$ & $\begin{array}{c}\text { Primary } \\
\text { Reference }\end{array}$ & $\begin{array}{l}\mathrm{Z} \text { and } \\
\text { Element }\end{array}$ & $\begin{array}{c}\text { Recommended } \\
\text { Value (Dry } \\
\text { Weight) } \\
\end{array}$ & $\begin{array}{c}\text { Compiled } \\
\text { in }\end{array}$ & $\begin{array}{c}\text { Primary } \\
\text { Reference }\end{array}$ \\
\hline $04 \mathrm{Be}$ & $1.0 \mathrm{E}-2$ & KS92 & $\mathrm{Ba} 84$ & $52 \mathrm{Te}$ & $2.5 \mathrm{E}-2$ & KS92 & $\mathrm{Ba} 84$ \\
\hline $06 \mathrm{C}$ & $7.0 \mathrm{E}-1$ & KS92 & & $53 \mathrm{I}$ & 4.0E-2 & $\mathrm{Sn} 94$ & $\mathrm{Sn} 94$ \\
\hline $07 \mathrm{~N}$ & $5.5 \mathrm{E}-2$ & KS92 & $\mathrm{Ba} 84$ & $55 \mathrm{Cs}$ & $4.6 \mathrm{E}-1$ & IAEA94 & Fr82 \\
\hline $09 \mathrm{~F}$ & $6.0 \mathrm{E}-2$ & KS92 & $\mathrm{Ba} 84$ & $56 \mathrm{Ba}$ & $1.5 \mathrm{E}-1$ & KS92 & $\mathrm{Ba} 84$ \\
\hline $11 \mathrm{Na}$ & $3.0 \mathrm{E}-1$ & IAEA94 & $\mathrm{Ng} 82 \mathrm{~b}$ & $57 \mathrm{La}$ & $5.2 \mathrm{E}-3$ & IAEA94 & Fr89 \\
\hline $12 \mathrm{Mg}$ & $1.0 \mathrm{E}+0$ & KS92 & $\mathrm{Ba} 84$ & $58 \mathrm{Ce}$ & $2.0 \mathrm{E}-2$ & $\mathrm{Ng} 82 \mathrm{~b}$ & $\mathrm{Ng} 82 \mathrm{~b}$ \\
\hline $14 \mathrm{Si}^{2}$ & $3.5 \mathrm{E}-1$ & $\mathrm{KS} 92$ & $\mathrm{Ba} 84$ & $59 \mathrm{Pr}$ & $2.0 \mathrm{E}-2$ & Based on $\mathrm{Ce}$ & $\mathrm{Ng} 82 \mathrm{~b}$ \\
\hline $15 \mathrm{P}$ & $3.5 \mathrm{E}+0$ & KS92 & $\mathrm{Ba} 84$ & $60 \mathrm{Nd}$ & $2.0 \mathrm{E}-2$ & Based on $\mathrm{Ce}$ & $\mathrm{Ng} 82 \mathrm{~b}$ \\
\hline $16 \mathrm{~S}$ & $1.5 \mathrm{E}+0$ & $\mathrm{KS} 92$ & $\mathrm{Ba} 84$ & $61 \mathrm{Pm}$ & $2.0 \mathrm{E}-2$ & Based on $\mathrm{Ce}$ & $\mathrm{Ng} 82 \mathrm{~b}$ \\
\hline $17 \mathrm{Cl}$ & $7.0 \mathrm{E}+1$ & KS92 & $\mathrm{Ba} 84$ & $62 \mathrm{Sm}$ & $2.0 \mathrm{E}-2$ & Based on $\mathrm{Ce}$ & $\mathrm{Ng} 82 \mathrm{~b}$ \\
\hline $19 \mathrm{~K}$ & $1.0 \mathrm{E}+0$ & KS92 & $\mathrm{Ba} 84$ & $63 \mathrm{Eu}$ & $2.0 \mathrm{E}-2$ & Based on $\mathrm{Ce}$ & $\mathrm{Ng} 82 \mathrm{~b}$ \\
\hline $20 \mathrm{Ca}$ & $3.5 \mathrm{E}+0$ & KS92 & $\mathrm{Ba} 84$ & $64 \mathrm{Gd}$ & $2.0 \mathrm{E}-2$ & Based on $\mathrm{Ce}$ & $\mathrm{Ng} 82 \mathrm{~b}$ \\
\hline $21 \mathrm{Sc}$ & $6.0 \mathrm{E}-3$ & $\mathrm{KS} 92$ & $\mathrm{Ba} 84$ & $65 \mathrm{~Tb}$ & $2.0 \mathrm{E}-2$ & Based on $\mathrm{Ce}$ & $\mathrm{Ng} 82 \mathrm{~b}$ \\
\hline $24 \mathrm{Cr}$ & $7.5 \mathrm{E}-3$ & KS92 & $\mathrm{Ba} 84$ & 66 Dy & $2.0 \mathrm{E}-2$ & Based on $\mathrm{Ce}$ & $\mathrm{Ng} 82 \mathrm{~b}$ \\
\hline $25 \mathrm{Mn}$ & 7.0E-1 & IAEA94 & Fr89 & $67 \mathrm{Ho}$ & $2.0 \mathrm{E}-2$ & Based on $\mathrm{Ce}$ & $\mathrm{Ng} 82 \mathrm{~b}$ \\
\hline $26 \mathrm{Fe}$ & $5.0 \mathrm{E}-2$ & CT83 & СТ83 & $68 \mathrm{Er}$ & $2.0 \mathrm{E}-2$ & Based on $\mathrm{Ce}$ & $\mathrm{Ng} 82 \mathrm{~b}$ \\
\hline $27 \mathrm{Co}$ & $2.3 \mathrm{E}-1$ & IAEA94 & Fr89 & $72 \mathrm{Hf}$ & $1.0 \mathrm{E}-3$ & Based on $\mathrm{Zr}$ & $\mathrm{Ng} 82 \mathrm{~b}$ \\
\hline $28 \mathrm{Ni}$ & $2.8 \mathrm{E}-1$ & KS92 & IUR89 & $73 \mathrm{Ta}$ & $2.5 \mathrm{E}-2$ & Based on $\mathrm{Nb}$ & СТ83 \\
\hline $29 \mathrm{Cu}$ & $4.0 \mathrm{E}-1$ & KS92 & $\mathrm{Ba} 84$ & $74 \mathrm{~W}$ & $3.0 \mathrm{E}+0$ & $\mathrm{Na} 88$ & NCRP86 \\
\hline $30 \mathrm{Zn}$ & $1.3 \mathrm{E}+0$ & IAEA94 & Fr89 & $75 \mathrm{Re}$ & $1.5 \mathrm{E}+0$ & KS92 & $\mathrm{Ba} 84$ \\
\hline $31 \mathrm{Ga}$ & $4.0 \mathrm{E}-3$ & Based on In & $\mathrm{Ba} 84$ & $76 \mathrm{Os}$ & $1.5 \mathrm{E}-2$ & KS92 & $\mathrm{Ba} 84$ \\
\hline $33 \mathrm{As}$ & 4.0E-2 & KS92 & $\mathrm{Ba} 84$ & $77 \mathrm{Ir}$ & $5.5 \mathrm{E}-2$ & KS92 & $\mathrm{Ba} 84$ \\
\hline $34 \mathrm{Se}$ & $2.5 \mathrm{E}-1$ & CT83 & СТ83 & $79 \mathrm{Au}$ & $1.0 \mathrm{E}-2$ & g.m. & g.m. \\
\hline $35 \mathrm{Br}$ & $1.5 \mathrm{E}+0$ & KS92 & $\mathrm{Fu} 78$ & $80 \mathrm{Hg}$ & $8.5 \mathrm{E}-1$ & g.m. & g.m. \\
\hline $37 \mathrm{Rb}$ & $9.0 \mathrm{E}-1$ & IAEA94 & $\mathrm{Ng} 82 \mathrm{~b}$ & $81 \mathrm{Tl}$ & $4.0 \mathrm{E}-3$ & Based on In & $\mathrm{Fu} 78$ \\
\hline $38 \mathrm{Sr}$ & $3.0 \mathrm{E}+0$ & IAEA94 & Fr82 & $82 \mathrm{~Pb}$ & $1.0 \mathrm{E}-2$ & IAEA94 & Fr89 \\
\hline $39 \mathrm{Y}$ & $1.0 \mathrm{E}-2$ & IAEA94 & $\mathrm{Ng} 82 \mathrm{~b}$ & $83 \mathrm{Bi}$ & $5.0 \mathrm{E}-1$ & IAEA94 & IAEA82 \\
\hline $40 \mathrm{Zr}$ & $1.0 \mathrm{E}-3$ & IAEA94 & Ng82b & $84 \mathrm{Po}$ & $1.2 \mathrm{E}-3$ & IAEA94 & Ho91 \\
\hline $41 \mathrm{Nb}$ & $2.5 \mathrm{E}-2$ & СТ83 & СТ83 & $88 \mathrm{Ra}$ & $4.9 \mathrm{E}-2$ & IAEA94 & Fr89 \\
\hline $42 \mathrm{Mo}$ & $8.0 \mathrm{E}-1$ & IAEA94 & $\mathrm{Ng} 82 \mathrm{~b}$ & $89 \mathrm{Ac}$ & 4.7E-4 & Based on Am & Fr82 \\
\hline $43 \mathrm{Tc}$ & $2.1 \mathrm{E}+2$ & IAEA94 & Fr89 & $90 \mathrm{Th}$ & $1.8 \mathrm{E}-3$ & IAEA94 & Fr89 \\
\hline $44 \mathrm{Ru}$ & $4.0 \mathrm{E}-2$ & IAEA94 & $\mathrm{Ng} 82 \mathrm{~b}$ & $91 \mathrm{~Pa}$ & 4.7E-4 & Based on Am & Fr82 \\
\hline $45 \mathrm{Rh}$ & $1.5 \mathrm{E}-1$ & KS92 & $\mathrm{Ba} 84$ & $92 \mathrm{U}$ & $8.3 \mathrm{E}-3$ & IAEA94 & Fr89 \\
\hline $46 \mathrm{Pd}$ & $1.5 \mathrm{E}-1$ & KS92 & $\mathrm{Ba} 84$ & $93 \mathrm{~Np}$ & $3.2 \mathrm{E}-2$ & IAEA94 & Fr82 \\
\hline $47 \mathrm{Ag}$ & 2.7E-4 & IAEA94 & Fr89 & $94 \mathrm{Pu}$ & $6.0 \mathrm{E}-5$ & IAEA94 & Fr82 \\
\hline $48 \mathrm{Cd}$ & $5.5 \mathrm{E}-1$ & KS92 & $\mathrm{Ba} 84$ & $95 \mathrm{Am}$ & 4.7E-4 & IAEA94 & Fr82 \\
\hline $49 \mathrm{In}$ & $4.0 \mathrm{E}-3$ & KS92 & $\mathrm{Fu} 78$ & $96 \mathrm{Cm}$ & 7.7E-4 & IAEA94 & Fr82 \\
\hline $50 \mathrm{Sn}$ & $3.0 \mathrm{E}-2$ & KS92 & $\mathrm{Fu} 78$ & $98 \mathrm{Cf}$ & 4.7E-4 & Based on Am & Fr82 \\
\hline $51 \mathrm{Sb}$ & $1.3 \mathrm{E}-4$ & KS92 & IUR89 & $98 \mathrm{Cf}$ & $2.5 \mathrm{E}-4$ & Based on Am & $\mathrm{Ba} 84$ \\
\hline
\end{tabular}


Table 2.3. Transfer Factors for Fruit (Bq/kg fruit per $\mathrm{Bq} / \mathrm{kg}$ soil)

\begin{tabular}{|c|c|c|c|c|c|c|c|}
\hline $\begin{array}{c}\mathrm{Z} \text { and } \\
\text { Element }\end{array}$ & $\begin{array}{c}\text { Recommended } \\
\text { Value (Dry } \\
\text { Weight) }\end{array}$ & $\begin{array}{c}\text { Compiled } \\
\text { in }\end{array}$ & $\begin{array}{c}\text { Primary } \\
\text { Reference }\end{array}$ & $\begin{array}{c}\mathrm{Z} \text { and } \\
\text { Element }\end{array}$ & $\begin{array}{c}\text { Recommended } \\
\text { Value (Dry } \\
\text { Weight) }\end{array}$ & $\begin{array}{c}\text { Compiled } \\
\text { in }\end{array}$ & $\begin{array}{c}\text { Primary } \\
\text { Reference }\end{array}$ \\
\hline $04 \mathrm{Be}$ & $1.5 \mathrm{E}-3$ & KS92 & Ba84 & $52 \mathrm{Te}$ & $4.0 \mathrm{E}-3$ & KS92 & $\mathrm{Ba} 84$ \\
\hline $06 \mathrm{C}$ & $7.0 \mathrm{E}-1$ & $\mathrm{Na} 88$ & $\mathrm{Na} 88$ & $53 \mathrm{I}$ & 4.0E-2 & Sn94 & Sn94 \\
\hline $07 \mathrm{~N}$ & $3.0 \mathrm{E}-2$ & g.m. & g.m. & $55 \mathrm{Cs}$ & $2.2 \mathrm{E}-1$ & IAEA94 & Fr89 \\
\hline $09 \mathrm{~F}$ & $6.0 \mathrm{E}-3$ & KS92 & $\mathrm{Ba} 84$ & $56 \mathrm{Ba}$ & $1.5 \mathrm{E}-2$ & KS92 & $\mathrm{Ba} 84$ \\
\hline $11 \mathrm{Na}$ & $3.0 \mathrm{E}-1$ & IAEA94 & $\mathrm{Ng} 82 \mathrm{~b}$ & $57 \mathrm{La}$ & $4.0 \mathrm{E}-3$ & KS92 & $\mathrm{Ba} 84$ \\
\hline $12 \mathrm{Mg}$ & $5.5 \mathrm{E}-1$ & KS92 & $\mathrm{Ba} 84$ & $58 \mathrm{Ce}$ & $2.0 \mathrm{E}-2$ & $\mathrm{Ng} 82 \mathrm{~b}$ & $\mathrm{Ng} 82 \mathrm{~b}$ \\
\hline $14 \mathrm{Si}$ & $7.0 \mathrm{E}-2$ & KS92 & $\mathrm{Ba} 84$ & $59 \operatorname{Pr}$ & $2.0 \mathrm{E}-2$ & Based on $\mathrm{Ce}$ & $\mathrm{Ng} 82 \mathrm{~b}$ \\
\hline $15 \mathrm{P}$ & $3.5 \mathrm{E}+0$ & KS92 & $\mathrm{Ba} 84$ & $60 \mathrm{Nd}$ & $2.0 \mathrm{E}-2$ & Based on $\mathrm{Ce}$ & $\mathrm{Ng} 82 \mathrm{~b}$ \\
\hline $16 \mathrm{~S}$ & $1.5 \mathrm{E}+0$ & KS92 & $\mathrm{Ba} 84$ & $61 \mathrm{Pm}$ & $2.0 \mathrm{E}-2$ & Based on $\mathrm{Ce}$ & $\mathrm{Ng} 82 \mathrm{~b}$ \\
\hline $17 \mathrm{Cl}$ & $7.0 \mathrm{E}+1$ & KS92 & $\mathrm{Ba} 84$ & $62 \mathrm{Sm}$ & $2.0 \mathrm{E}-2$ & Based on $\mathrm{Ce}$ & $\mathrm{Ng} 82 \mathrm{~b}$ \\
\hline $19 \mathrm{~K}$ & $5.5 \mathrm{E}-1$ & KS92 & $\mathrm{Ba} 84$ & $63 \mathrm{Eu}$ & $2.0 \mathrm{E}-2$ & Based on $\mathrm{Ce}$ & $\mathrm{Ng} 82 \mathrm{~b}$ \\
\hline $20 \mathrm{Ca}$ & $3.5 \mathrm{E}-1$ & KS92 & Ba84 & $64 \mathrm{Gd}$ & $2.0 \mathrm{E}-2$ & Based on $\mathrm{Ce}$ & $\mathrm{Ng} 82 \mathrm{~b}$ \\
\hline $21 \mathrm{Sc}$ & $1.0 \mathrm{E}-3$ & KS92 & Ba84 & $65 \mathrm{~Tb}$ & $2.0 \mathrm{E}-2$ & Based on $\mathrm{Ce}$ & $\mathrm{Ng} 82 \mathrm{~b}$ \\
\hline $24 \mathrm{Cr}$ & $4.5 \mathrm{E}-3$ & KS92 & Ba84 & 66 Dy & $2.0 \mathrm{E}-2$ & Based on $\mathrm{Ce}$ & $\mathrm{Ng} 82 \mathrm{~b}$ \\
\hline $25 \mathrm{Mn}$ & $5.0 \mathrm{E}-2$ & KS92 & $\mathrm{Ba} 84$ & $67 \mathrm{Ho}$ & $2.0 \mathrm{E}-2$ & Based on $\mathrm{Ce}$ & $\mathrm{Ng} 82 \mathrm{~b}$ \\
\hline $26 \mathrm{Fe}$ & $5.0 \mathrm{E}-2$ & CT83 & СТ83 & $68 \mathrm{Er}$ & $2.0 \mathrm{E}-2$ & Based on $\mathrm{Ce}$ & $\mathrm{Ng} 82 \mathrm{~b}$ \\
\hline $27 \mathrm{Co}$ & $7.0 \mathrm{E}-3$ & KS92 & $\mathrm{Ba} 84$ & $72 \mathrm{Hf}$ & $1.0 \mathrm{E}-3$ & Based on $\mathrm{Zr}$ & $\mathrm{Ng} 82 \mathrm{~b}$ \\
\hline $28 \mathrm{Ni}$ & $6.0 \mathrm{E}-2$ & KS92 & $\mathrm{Ba} 84$ & $73 \mathrm{Ta}$ & $2.5 \mathrm{E}-2$ & Based on $\mathrm{Nb}$ & CT83 \\
\hline $29 \mathrm{Cu}$ & $2.5 \mathrm{E}-1$ & KS92 & Ba84 & $74 \mathrm{~W}$ & $3.0 \mathrm{E}+0$ & NCRP96 & NCRP96 \\
\hline $30 \mathrm{Zn}$ & $9.0 \mathrm{E}-1$ & KS92 & Ba84 & $75 \mathrm{Re}$ & $3.5 \mathrm{E}-1$ & KS92 & $\mathrm{Ba} 84$ \\
\hline $31 \mathrm{Ga}$ & $4.0 \mathrm{E}-4$ & Based on In & $\mathrm{Ba} 84$ & $76 \mathrm{Os}$ & $4.5 \mathrm{E}-2$ & g.m. & g.m. \\
\hline $33 \mathrm{As}$ & $6.0 \mathrm{E}-3$ & KS92 & $\mathrm{Ba} 84$ & $77 \mathrm{Ir}$ & $1.5 \mathrm{E}-2$ & KS92 & $\mathrm{Ba} 84$ \\
\hline $34 \mathrm{Se}$ & $5.0 \mathrm{E}-2$ & CT83 & СТ83 & $79 \mathrm{Au}$ & $1.4 \mathrm{E}-2$ & g.m. & g.m. \\
\hline $35 \mathrm{Br}$ & $1.5 \mathrm{E}+0$ & KS92 & $\mathrm{Fu} 78$ & $80 \mathrm{Hg}$ & $3.7 \mathrm{E}-1$ & g.m. & g.m. \\
\hline $37 \mathrm{Rb}$ & $9.0 \mathrm{E}-1$ & IAEA94 & $\mathrm{Ng} 82 \mathrm{~b}$ & $81 \mathrm{Tl}$ & $4.0 \mathrm{E}-4$ & KS92 & $\mathrm{Ba} 84$ \\
\hline $38 \mathrm{Sr}$ & $2.0 \mathrm{E}-1$ & IAEA94 & Fr82 & $82 \mathrm{~Pb}$ & $1.0 \mathrm{E}-2$ & IAEA94 & Fr89 \\
\hline $39 \mathrm{Y}$ & $1.0 \mathrm{E}-2$ & IAEA94 & $\mathrm{Ng} 82 \mathrm{~b}$ & $83 \mathrm{Bi}$ & $5.0 \mathrm{E}-1$ & IAEA94 & IAEA82 \\
\hline $40 \mathrm{Zr}$ & $1.0 \mathrm{E}-3$ & IAEA94 & $\mathrm{Ng} 82 \mathrm{~b}$ & $84 \mathrm{Po}$ & $1.2 \mathrm{E}-3$ & IAEA94 & Ho91 \\
\hline $41 \mathrm{Nb}$ & $2.5 \mathrm{E}-2$ & СТ83 & СТ83 & $88 \mathrm{Ra}$ & $6.1 \mathrm{E}-3$ & IAEA94 & Fr89 \\
\hline $42 \mathrm{Mo}$ & $5.0 \mathrm{E}-2$ & СТ83 & СТ83 & $89 \mathrm{Ac}$ & $2.5 \mathrm{E}-4$ & Based on Am & Ba84 \\
\hline $43 \mathrm{Tc}$ & $1.5 \mathrm{E}+0$ & KS92 & $\mathrm{Ba} 84$ & $90 \mathrm{Th}$ & $2.5 \mathrm{E}-4$ & Based on Am & $\mathrm{Ba} 84$ \\
\hline $44 \mathrm{Ru}$ & $4.0 \mathrm{E}-2$ & IAEA94 & $\mathrm{Ng} 82 \mathrm{~b}$ & $91 \mathrm{~Pa}$ & $2.5 \mathrm{E}-4$ & Based on Am & $\mathrm{Ba} 84$ \\
\hline $45 \mathrm{Rh}$ & $4.0 \mathrm{E}-2$ & KS92 & $\mathrm{Ba} 84$ & $92 \mathrm{U}$ & $4.0 \mathrm{E}-3$ & KS92 & $\mathrm{Ba} 84$ \\
\hline $46 \mathrm{Pd}$ & $4.0 \mathrm{E}-2$ & KS92 & Ba84 & $93 \mathrm{~Np}$ & $1.0 \mathrm{E}-2$ & KS92 & $\mathrm{Ba} 84$ \\
\hline $47 \mathrm{Ag}$ & $8.0 \mathrm{E}-4$ & IAEA94 & Fr89 & $94 \mathrm{Pu}$ & $4.5 \mathrm{E}-5$ & KS92 & $\mathrm{Ba} 84$ \\
\hline $48 \mathrm{Cd}$ & $1.5 \mathrm{E}-1$ & KS92 & $\mathrm{Ba} 84$ & $95 \mathrm{Am}$ & $2.5 \mathrm{E}-4$ & KS92 & $\mathrm{Ba} 84$ \\
\hline 49 In & $4.0 \mathrm{E}-4$ & KS92 & $\mathrm{Fu} 78$ & $96 \mathrm{Cm}$ & $1.5 \mathrm{E}-5$ & KS92 & $\mathrm{Ba} 84$ \\
\hline $50 \mathrm{Sn}$ & $6.0 \mathrm{E}-3$ & KS92 & $\mathrm{Fu} 78$ & $98 \mathrm{Cf}$ & $2.5 \mathrm{E}-4$ & Based on Am & $\mathrm{Ba} 84$ \\
\hline $51 \mathrm{Sb}$ & $8.0 \mathrm{E}-5$ & KS92 & IUR89 & & & & \\
\hline
\end{tabular}


Table 2.4. Transfer Factors for Root Vegetables (Bq/kg root vegetable per $\mathrm{Bq} / \mathrm{kg}$ soil)

\begin{tabular}{|c|c|c|c|c|c|c|c|}
\hline $\begin{array}{c}\mathrm{Z} \text { and } \\
\text { Element }\end{array}$ & $\begin{array}{c}\text { Recommended } \\
\text { Value (Dry } \\
\text { Weight) }\end{array}$ & Compiled in & $\begin{array}{c}\text { Primary } \\
\text { Reference }\end{array}$ & $\begin{array}{c}\mathrm{Z} \text { and } \\
\text { Element }\end{array}$ & $\begin{array}{c}\text { Recommended } \\
\text { Value (Dry } \\
\text { Weight) }\end{array}$ & Compiled in & $\begin{array}{c}\text { Primary } \\
\text { Reference }\end{array}$ \\
\hline $04 \mathrm{Be}$ & $1.5 \mathrm{E}-3$ & KS92 & $\mathrm{Ba} 84$ & $52 \mathrm{Te}$ & $4.0 \mathrm{E}-3$ & KS92 & $\mathrm{Ba} 84$ \\
\hline $06 \mathrm{C}$ & $7.0 \mathrm{E}-1$ & $\mathrm{Na} 88$ & $\mathrm{Na} 88$ & $53 \mathrm{I}$ & 4.0E-2 & $\operatorname{Sn} 94$ & Sn94 \\
\hline $07 \mathrm{~N}$ & $4.9 \mathrm{E}-2$ & KS92 & $\mathrm{Ba} 84$ & $55 \mathrm{Cs}$ & $1.3 \mathrm{E}-1$ & IAEA94 & Fr82 \\
\hline $09 \mathrm{~F}$ & $6.0 \mathrm{E}-3$ & KS92 & $\mathrm{Ba} 84$ & $56 \mathrm{Ba}$ & $1.5 \mathrm{E}-2$ & KS92 & $\mathrm{Ba} 84$ \\
\hline $11 \mathrm{Na}$ & $3.0 \mathrm{E}-1$ & IAEA94 & $\mathrm{Ng} 82 \mathrm{~b}$ & $57 \mathrm{La}$ & $3.5 \mathrm{E}-4$ & IAEA94 & Fr89 \\
\hline $12 \mathrm{Mg}$ & $5.5 \mathrm{E}-1$ & KS92 & $\mathrm{Ba} 84$ & $58 \mathrm{Ce}$ & $2.0 \mathrm{E}-2$ & $\mathrm{Ng} 82 \mathrm{~b}$ & $\mathrm{Ng} 82 \mathrm{~b}$ \\
\hline $14 \mathrm{Si}$ & 7.0E-2 & KS92 & Ba84 & $59 \operatorname{Pr}$ & $2.0 \mathrm{E}-2$ & Based on $\mathrm{Ce}$ & $\mathrm{Ng} 82 \mathrm{~b}$ \\
\hline $15 \mathrm{P}$ & $3.5 \mathrm{E}+0$ & KS92 & $\mathrm{Ba} 84$ & $60 \mathrm{Nd}$ & $2.0 \mathrm{E}-2$ & Based on $\mathrm{Ce}$ & $\mathrm{Ng} 82 \mathrm{~b}$ \\
\hline $16 \mathrm{~S}$ & $1.5 \mathrm{E}+0$ & KS92 & $\mathrm{Ba} 84$ & $61 \mathrm{Pm}$ & $2.0 \mathrm{E}-2$ & Based on $\mathrm{Ce}$ & $\mathrm{Ng} 82 \mathrm{~b}$ \\
\hline $17 \mathrm{Cl}$ & $7.0 \mathrm{E}+1$ & KS92 & Ba84 & $62 \mathrm{Sm}$ & $2.0 \mathrm{E}-2$ & Based on $\mathrm{Ce}$ & $\mathrm{Ng} 82 \mathrm{~b}$ \\
\hline $19 \mathrm{~K}$ & $5.5 \mathrm{E}-1$ & KS92 & $\mathrm{Ba} 84$ & $63 \mathrm{Eu}$ & $2.0 \mathrm{E}-2$ & Based on $\mathrm{Ce}$ & $\mathrm{Ng} 82 \mathrm{~b}$ \\
\hline $20 \mathrm{Ca}$ & $3.5 \mathrm{E}-1$ & $\mathrm{KS} 92$ & Ba84 & $64 \mathrm{Gd}$ & $2.0 \mathrm{E}-2$ & Based on $\mathrm{Ce}$ & $\mathrm{Ng} 82 \mathrm{~b}$ \\
\hline $21 \mathrm{Sc}$ & $1.0 \mathrm{E}-3$ & $\mathrm{KS} 92$ & Ba84 & $65 \mathrm{~Tb}$ & $2.0 \mathrm{E}-2$ & Based on $\mathrm{Ce}$ & $\mathrm{Ng} 82 \mathrm{~b}$ \\
\hline $24 \mathrm{Cr}$ & $4.5 \mathrm{E}-3$ & $\mathrm{KS} 92$ & Ba84 & 66 Dy & $2.0 \mathrm{E}-2$ & Based on $\mathrm{Ce}$ & $\mathrm{Ng} 82 \mathrm{~b}$ \\
\hline $25 \mathrm{Mn}$ & $2.0 \mathrm{E}-1$ & IAEA94 & Fr89 & $67 \mathrm{Ho}$ & $2.0 \mathrm{E}-2$ & Based on $\mathrm{Ce}$ & $\mathrm{Ng} 82 \mathrm{~b}$ \\
\hline $26 \mathrm{Fe}$ & $5.0 \mathrm{E}-2$ & СТ83 & СТ83 & $68 \mathrm{Er}$ & $2.0 \mathrm{E}-2$ & Based on $\mathrm{Ce}$ & $\mathrm{Ng} 82 \mathrm{~b}$ \\
\hline $27 \mathrm{Co}$ & $6.7 \mathrm{E}-2$ & IAEA94 & Fr89 & $72 \mathrm{Hf}$ & $1.0 \mathrm{E}-3$ & Based on $\mathrm{Zr}$ & $\mathrm{Ng} 82 \mathrm{~b}$ \\
\hline $28 \mathrm{Ni}$ & $6.0 \mathrm{E}-2$ & KS92 & $\mathrm{Ba} 84$ & $73 \mathrm{Ta}$ & $2.5 \mathrm{E}-2$ & Based on $\mathrm{Nb}$ & CT83 \\
\hline $29 \mathrm{Cu}$ & $2.5 \mathrm{E}-1$ & KS92 & Ba84 & $74 \mathrm{~W}$ & $3.0 \mathrm{E}+0$ & $\mathrm{Na} 88$ & NCRP86 \\
\hline $30 \mathrm{Zn}$ & $3.5 \mathrm{E}-1$ & IAEA94 & Fr89 & $75 \mathrm{Re}$ & $3.5 \mathrm{E}-1$ & KS92 & Ba84 \\
\hline $31 \mathrm{Ga}$ & $4.0 \mathrm{E}-4$ & Based on In & Ba84 & $76 \mathrm{Os}$ & $3.5 \mathrm{E}-3$ & $\mathrm{KS} 92$ & $\mathrm{Ba} 84$ \\
\hline $33 \mathrm{As}$ & $6.0 \mathrm{E}-3$ & KS92 & Ba84 & $77 \mathrm{Ir}$ & $1.5 \mathrm{E}-2$ & KS92 & $\mathrm{Ba} 84$ \\
\hline $34 \mathrm{Se}$ & $5.0 \mathrm{E}-2$ & СТ83 & СТ83 & $79 \mathrm{Au}$ & $1.8 \mathrm{E}-2$ & g.m. & g.m. \\
\hline $35 \mathrm{Br}$ & $1.5 \mathrm{E}+0$ & KS92 & $\mathrm{Fu} 78$ & $80 \mathrm{Hg}$ & $2.0 \mathrm{E}-1$ & KS92 & $\mathrm{Ba} 84$ \\
\hline $37 \mathrm{Rb}$ & $9.0 \mathrm{E}-1$ & IAEA94 & $\mathrm{Ng} 82 \mathrm{~b}$ & $81 \mathrm{Tl}$ & $4.0 \mathrm{E}-4$ & Based on In & Fu78 \\
\hline $38 \mathrm{Sr}$ & $5.0 \mathrm{E}-1$ & IAEA94 & Fr82 & $82 \mathrm{~Pb}$ & $6.0 \mathrm{E}-3$ & IAEA94 & Fr89 \\
\hline $39 \mathrm{Y}$ & $1.0 \mathrm{E}-2$ & IAEA94 & $\mathrm{Ng} 82 \mathrm{~b}$ & $83 \mathrm{Bi}$ & $5.0 \mathrm{E}-1$ & IAEA94 & IAEA82 \\
\hline $40 \mathrm{Zr}$ & $1.0 \mathrm{E}-3$ & IAEA94 & $\mathrm{Ng} 82 \mathrm{~b}$ & $84 \mathrm{Po}$ & $7.0 \mathrm{E}-3$ & IAEA94 & HW91 \\
\hline $41 \mathrm{Nb}$ & $2.5 \mathrm{E}-2$ & СТ83 & СТ83 & $88 \mathrm{Ra}$ & $2.0 \mathrm{E}-3$ & IAEA94 & Fr89 \\
\hline $42 \mathrm{Mo}$ & $8.0 \mathrm{E}-1$ & IAEA94 & $\mathrm{Ng} 82 \mathrm{~b}$ & $89 \mathrm{Ac}$ & $3.5 \mathrm{E}-4$ & Based on Am & Fr82 \\
\hline $43 \mathrm{Tc}$ & $2.4 \mathrm{E}-1$ & IAEA94 & Fr89 & $90 \mathrm{Th}$ & $3.3 \mathrm{E}-4$ & IAEA94 & Fr89 \\
\hline $44 \mathrm{Ru}$ & $4.0 \mathrm{E}-2$ & IAEA94 & $\mathrm{Ng} 82 \mathrm{~b}$ & $91 \mathrm{~Pa}$ & $3.5 \mathrm{E}-4$ & Based on Am & Fr82 \\
\hline $45 \mathrm{Rh}$ & $4.0 \mathrm{E}-2$ & KS92 & $\mathrm{Ba} 84$ & $92 \mathrm{U}$ & $1.2 \mathrm{E}-2$ & IAEA94 & Fr89 \\
\hline $46 \mathrm{Pd}$ & $4.0 \mathrm{E}-2$ & KS92 & Ba84 & $93 \mathrm{~Np}$ & $1.3 \mathrm{E}-2$ & IAEA94 & Fr82 \\
\hline $47 \mathrm{Ag}$ & $1.3 \mathrm{E}-3$ & IAEA94 & Fr89 & $94 \mathrm{Pu}$ & $1.1 \mathrm{E}-3$ & IAEA94 & Fr82 \\
\hline $48 \mathrm{Cd}$ & $1.5 \mathrm{E}-1$ & KS92 & $\mathrm{Ba} 84$ & $95 \mathrm{Am}$ & $3.5 \mathrm{E}-4$ & IAEA94 & Fr82 \\
\hline 49 In & $4.0 \mathrm{E}-4$ & KS92 & $\mathrm{Fu} 78$ & $96 \mathrm{Cm}$ & $4.3 \mathrm{E}-4$ & IAEA94 & Fr82 \\
\hline $50 \mathrm{Sn}$ & $6.0 \mathrm{E}-3$ & KS92 & $\mathrm{Fu} 78$ & $98 \mathrm{Cf}$ & $3.5 \mathrm{E}-4$ & Based on Am & Fr82 \\
\hline $51 \mathrm{Sb}$ & $5.6 \mathrm{E}-4$ & IAEA94 & Fr89 & & & & \\
\hline
\end{tabular}


Table 2.5. Transfer Factors for Grain (Bq/kg grain per Bq/kg soil)

\begin{tabular}{|c|c|c|c|c|c|c|c|}
\hline $\begin{array}{c}\mathrm{Z} \text { and } \\
\text { Element }\end{array}$ & $\begin{array}{c}\text { Recommended } \\
\text { Value (Dry } \\
\text { Weight) }\end{array}$ & $\begin{array}{c}\text { Compiled } \\
\text { in }\end{array}$ & $\begin{array}{c}\text { Primary } \\
\text { Reference }\end{array}$ & $\begin{array}{c}\mathrm{Z} \text { and } \\
\text { Element }\end{array}$ & $\begin{array}{c}\text { Recommended } \\
\text { Value (Dry } \\
\text { Weight) }\end{array}$ & $\begin{array}{c}\text { Compiled } \\
\text { in }\end{array}$ & $\begin{array}{c}\text { Primary } \\
\text { Reference }\end{array}$ \\
\hline $04 \mathrm{Be}$ & $2.0 \mathrm{E}-3$ & g.m. & g.m. & $52 \mathrm{Te}$ & $4.0 \mathrm{E}-3$ & KS92 & $\mathrm{Ba} 84$ \\
\hline $06 \mathrm{C}$ & $7.0 \mathrm{E}-1$ & $\mathrm{Na} 88$ & $\mathrm{Na} 88$ & $53 \mathrm{I}$ & $4.0 \mathrm{E}-2$ & Sn94 & Sn94 \\
\hline $07 \mathrm{~N}$ & $1.3 \mathrm{E}-1$ & g.m. & g.m. & $55 \mathrm{Cs}$ & $2.6 \mathrm{E}-2$ & IAEA94 & Fr82 \\
\hline $09 \mathrm{~F}$ & $6.0 \mathrm{E}-3$ & $\mathrm{KS} 92$ & $\mathrm{Ba} 84$ & $56 \mathrm{Ba}$ & $1.5 \mathrm{E}-2$ & KS92 & Ba84 \\
\hline $11 \mathrm{Na}$ & $3.0 \mathrm{E}-1$ & IAEA94 & $\mathrm{Ng} 82 \mathrm{~b}$ & $57 \mathrm{La}$ & $4.0 \mathrm{E}-3$ & KS92 & Ba84 \\
\hline $12 \mathrm{Mg}$ & $5.5 \mathrm{E}-1$ & KS92 & $\mathrm{Ba} 84$ & $58 \mathrm{Ce}$ & $2.0 \mathrm{E}-2$ & $\mathrm{Ng} 82 \mathrm{~b}$ & $\mathrm{Ng} 82 \mathrm{~b}$ \\
\hline $14 \mathrm{Si}$ & 7.0E-2 & KS92 & $\mathrm{Ba} 84$ & $59 \mathrm{Pr}$ & $2.0 \mathrm{E}-2$ & Based on $\mathrm{Ce}$ & $\mathrm{Ng} 82 \mathrm{~b}$ \\
\hline $15 \mathrm{P}$ & $3.5 \mathrm{E}+0$ & KS92 & $\mathrm{Ba} 84$ & $60 \mathrm{Nd}$ & $2.0 \mathrm{E}-2$ & Based on $\mathrm{Ce}$ & $\mathrm{Ng} 82 \mathrm{~b}$ \\
\hline $16 \mathrm{~S}$ & $1.5 \mathrm{E}+0$ & KS92 & Ba84 & $61 \mathrm{Pm}$ & $2.0 \mathrm{E}-2$ & Based on $\mathrm{Ce}$ & $\mathrm{Ng} 82 \mathrm{~b}$ \\
\hline $17 \mathrm{Cl}$ & $7.0 \mathrm{E}+1$ & KS92 & $\mathrm{Ba} 84$ & $62 \mathrm{Sm}$ & $2.0 \mathrm{E}-2$ & Based on $\mathrm{Ce}$ & $\mathrm{Ng} 82 \mathrm{~b}$ \\
\hline $19 \mathrm{~K}$ & $5.5 \mathrm{E}-1$ & KS92 & $\mathrm{Ba} 84$ & $63 \mathrm{Eu}$ & $2.0 \mathrm{E}-2$ & Based on $\mathrm{Ce}$ & $\mathrm{Ng} 82 \mathrm{~b}$ \\
\hline $20 \mathrm{Ca}$ & $3.5 \mathrm{E}-1$ & KS92 & $\mathrm{Ba} 84$ & $64 \mathrm{Gd}$ & $2.0 \mathrm{E}-2$ & Based on $\mathrm{Ce}$ & $\mathrm{Ng} 82 \mathrm{~b}$ \\
\hline $21 \mathrm{Sc}$ & $1.0 \mathrm{E}-3$ & KS92 & $\mathrm{Ba} 84$ & $65 \mathrm{~Tb}$ & $2.0 \mathrm{E}-2$ & Based on $\mathrm{Ce}$ & $\mathrm{Ng} 82 \mathrm{~b}$ \\
\hline $24 \mathrm{Cr}$ & $4.5 \mathrm{E}-3$ & KS92 & $\mathrm{Ba} 84$ & 66 Dy & $2.0 \mathrm{E}-2$ & Based on $\mathrm{Ce}$ & $\mathrm{Ng} 82 \mathrm{~b}$ \\
\hline $25 \mathrm{Mn}$ & $3.0 \mathrm{E}-1$ & IAEA94 & AEA94 & $67 \mathrm{Ho}$ & $2.0 \mathrm{E}-2$ & Based on $\mathrm{Ce}$ & $\mathrm{Ng} 82 \mathrm{~b}$ \\
\hline $26 \mathrm{Fe}$ & $5.0 \mathrm{E}-2$ & CT83 & CT83 & $68 \mathrm{Er}$ & $2.0 \mathrm{E}-2$ & Based on $\mathrm{Ce}$ & $\mathrm{Ng} 82 \mathrm{~b}$ \\
\hline $27 \mathrm{Co}$ & $3.7 \mathrm{E}-3$ & IAEA94 & Fr89 & $72 \mathrm{Hf}$ & $1.0 \mathrm{E}-3$ & Based on $\mathrm{Zr}$ & $\mathrm{Ng} 82 \mathrm{~b}$ \\
\hline $28 \mathrm{Ni}$ & $3.0 \mathrm{E}-2$ & IAEA94 & Fr89 & $73 \mathrm{Ta}$ & $2.5 \mathrm{E}-2$ & Based on $\mathrm{Nb}$ & СТ83 \\
\hline $29 \mathrm{Cu}$ & $2.5 \mathrm{E}-1$ & KS92 & Ba84 & $74 \mathrm{~W}$ & $3.0 \mathrm{E}+0$ & NCRP96 & NCRP96 \\
\hline $30 \mathrm{Zn}$ & $1.6 \mathrm{E}+0$ & IAEA94 & Fr89 & $75 \mathrm{Re}$ & $3.5 \mathrm{E}-1$ & KS92 & Ba84 \\
\hline $31 \mathrm{Ga}$ & 4.0E-4 & Based on In & $\mathrm{Ba} 84$ & $76 \mathrm{Os}$ & $3.5 \mathrm{E}-3$ & KS92 & $\mathrm{Ba} 84$ \\
\hline $33 \mathrm{As}$ & $6.0 \mathrm{E}-3$ & KS92 & $\mathrm{Ba} 84$ & $77 \mathrm{Ir}$ & $1.5 \mathrm{E}-2$ & KS92 & $\mathrm{Ba} 84$ \\
\hline $34 \mathrm{Se}$ & $2.5 \mathrm{E}-1$ & СТ83 & СТ83 & $79 \mathrm{Au}$ & $2.5 \mathrm{E}-1$ & Based on $\mathrm{Cu}$ & Ba84 \\
\hline $35 \mathrm{Br}$ & $1.5 \mathrm{E}+0$ & KS92 & $\mathrm{Fu} 78$ & $80 \mathrm{Hg}$ & $4.9 \mathrm{E}-1$ & g.m. & g.m. \\
\hline $37 \mathrm{Rb}$ & $9.0 \mathrm{E}-1$ & IAEA94 & $\mathrm{Ng} 82 \mathrm{~b}$ & $81 \mathrm{Tl}$ & $4.0 \mathrm{E}-4$ & KS92 & $\mathrm{Ba} 84$ \\
\hline $38 \mathrm{Sr}$ & $2.1 \mathrm{E}-1$ & IAEA94 & Fr82 & $82 \mathrm{~Pb}$ & $4.7 \mathrm{E}-3$ & IAEA94 & Fr89 \\
\hline $39 \mathrm{Y}$ & $1.0 \mathrm{E}-2$ & IAEA94 & $\mathrm{Ng} 82 \mathrm{~b}$ & $83 \mathrm{Bi}$ & $5.0 \mathrm{E}-1$ & IAEA94 & IAEA82 \\
\hline $40 \mathrm{Zr}$ & $1.0 \mathrm{E}-3$ & IAEA94 & $\mathrm{Ng} 82 \mathrm{~b}$ & $84 \mathrm{Po}$ & $2.3 \mathrm{E}-3$ & IAEA94 & HW91 \\
\hline $41 \mathrm{Nb}$ & $2.5 \mathrm{E}-2$ & СТ83 & СТ83 & $88 \mathrm{Ra}$ & $1.2 \mathrm{E}-3$ & IAEA94 & Fr89 \\
\hline $42 \mathrm{Mo}$ & $8.0 \mathrm{E}-1$ & IAEA94 & $\mathrm{Ng} 82 \mathrm{~b}$ & $89 \mathrm{Ac}$ & $2.2 \mathrm{E}-5$ & Based on Am & Fr82 \\
\hline $43 \mathrm{Tc}$ & 7.3E-1 & IAEA94 & Fr89 & $90 \mathrm{Th}$ & $3.4 \mathrm{E}-5$ & KS92 & IUR89 \\
\hline $44 \mathrm{Ru}$ & $5.0 \mathrm{E}-3$ & IAEA94 & Fr89 & $91 \mathrm{~Pa}$ & $2.2 \mathrm{E}-5$ & Based on Am & Fr82 \\
\hline $45 \mathrm{Rh}$ & $4.0 \mathrm{E}-2$ & KS92 & $\mathrm{Ba} 84$ & $92 \mathrm{U}$ & $1.3 \mathrm{E}-3$ & IAEA94 & Bi91 \\
\hline $46 \mathrm{Pd}$ & $4.0 \mathrm{E}-2$ & KS92 & $\mathrm{Ba} 84$ & $93 \mathrm{~Np}$ & $2.7 \mathrm{E}-3$ & IAEA94 & Fr82 \\
\hline $47 \mathrm{Ag}$ & $2.5 \mathrm{E}-1$ & Based on $\mathrm{Cu}$ & $\mathrm{Ba} 84$ & $94 \mathrm{Pu}$ & $8.6 \mathrm{E}-6$ & IAEA94 & Fr82 \\
\hline $48 \mathrm{Cd}$ & $1.5 \mathrm{E}-1$ & KS92 & $\mathrm{Ba} 84$ & $95 \mathrm{Am}$ & $2.2 \mathrm{E}-5$ & IAEA94 & Fr82 \\
\hline $49 \mathrm{In}$ & 4.0E-4 & KS92 & $\mathrm{Fu} 78$ & $96 \mathrm{Cm}$ & $2.1 \mathrm{E}-5$ & IAEA94 & Fr82 \\
\hline $50 \mathrm{Sn}$ & $6.0 \mathrm{E}-3$ & KS92 & $\mathrm{Fu} 78$ & $98 \mathrm{Cf}$ & $2.2 \mathrm{E}-5$ & Based on Am & Fr82 \\
\hline $51 \mathrm{Sb}$ & $3.0 \mathrm{E}-2$ & KS92 & $\mathrm{Fu} 78$ & & & & \\
\hline
\end{tabular}


Table 2.6. Transfer Factors for Beef (Bq/kg muscle per Bq/d intake)

\begin{tabular}{|c|c|c|c|c|c|c|c|}
\hline $\begin{array}{c}\mathrm{Z} \text { and } \\
\text { Element }\end{array}$ & $\begin{array}{l}\text { Recommended } \\
\text { Value (Wet } \\
\text { Weight) }\end{array}$ & $\begin{array}{c}\text { Compiled } \\
\text { in }\end{array}$ & $\begin{array}{c}\text { Primary } \\
\text { Reference }\end{array}$ & $\begin{array}{c}\mathrm{Z} \text { and } \\
\text { Element }\end{array}$ & $\begin{array}{c}\text { Recommended } \\
\text { Value (Wet } \\
\text { Weight) }\end{array}$ & $\begin{array}{c}\text { Compiled } \\
\text { in }\end{array}$ & $\begin{array}{c}\text { Primary } \\
\text { Reference }\end{array}$ \\
\hline $04 \mathrm{Be}$ & $1.0 \mathrm{E}-3$ & KS92 & Ng68 & $52 \mathrm{Te}$ & $7.0 \mathrm{E}-3$ & IAEA94 & Jo88 \\
\hline $07 \mathrm{~N}$ & $7.5 \mathrm{E}-2$ & KS92 & Ng68 & $53 \mathrm{I}$ & 4.0E-2 & IAEA94 & Bi89 \\
\hline $09 \mathrm{~F}$ & $1.5 \mathrm{E}-1$ & KS92 & Ng68 & $55 \mathrm{Cs}$ & $5.0 \mathrm{E}-2$ & IAEA94 & Co90 \\
\hline $11 \mathrm{Na}$ & $8.0 \mathrm{E}-2$ & IAEA94 & $\mathrm{Ng} 82 \mathrm{a}$ & $56 \mathrm{Ba}$ & $2.0 \mathrm{E}-4$ & IAEA94 & Jo88 \\
\hline $12 \mathrm{Mg}$ & $2.0 \mathrm{E}-2$ & IAEA94 & $\mathrm{Ng} 82 \mathrm{a}$ & $57 \mathrm{La}$ & $2.0 \mathrm{E}-3$ & NCRP96 & NCRP96 \\
\hline $14 \mathrm{Si}$ & $4.0 \mathrm{E}-5$ & KS92 & Ng68 & $58 \mathrm{Ce}$ & $2.0 \mathrm{E}-5$ & IAEA94 & CEC87 \\
\hline $15 \mathrm{P}$ & $5.0 \mathrm{E}-2$ & IAEA94 & $\mathrm{Ng} 82 \mathrm{a}$ & $59 \operatorname{Pr}$ & $2.0 \mathrm{E}-5$ & Based on $\mathrm{Ce}$ & CEC87 \\
\hline $16 \mathrm{~S}$ & $2.0 \mathrm{E}-1$ & $\mathrm{Na} 88$ & IAEA 87 & $60 \mathrm{Nd}$ & $2.0 \mathrm{E}-5$ & Based on $\mathrm{Ce}$ & CEC87 \\
\hline $17 \mathrm{Cl}$ & $2.0 \mathrm{E}-2$ & IAEA94 & Bi89 & $61 \mathrm{Pm}$ & $2.0 \mathrm{E}-5$ & Based on $\mathrm{Ce}$ & CEC87 \\
\hline $19 \mathrm{~K}$ & $2.0 \mathrm{E}-2$ & IAEA94 & $\mathrm{Ng} 82 \mathrm{a}$ & $62 \mathrm{Sm}$ & $2.0 \mathrm{E}-5$ & Based on $\mathrm{Ce}$ & CEC87 \\
\hline $20 \mathrm{Ca}$ & $2.0 \mathrm{E}-3$ & IAEA94 & $\mathrm{Ng} 82 \mathrm{a}$ & $63 \mathrm{Eu}$ & $2.0 \mathrm{E}-5$ & Based on $\mathrm{Ce}$ & CEC87 \\
\hline $21 \mathrm{Sc}$ & $1.5 \mathrm{E}-2$ & $\mathrm{KS} 92$ & $\mathrm{Ng} 68$ & $64 \mathrm{Gd}$ & $2.0 \mathrm{E}-5$ & Based on $\mathrm{Ce}$ & CEC87 \\
\hline $24 \mathrm{Cr}$ & $9.0 \mathrm{E}-3$ & IAEA94 & $\mathrm{Ng} 82 \mathrm{a}$ & $65 \mathrm{~Tb}$ & $2.0 \mathrm{E}-5$ & Based on $\mathrm{Ce}$ & CEC87 \\
\hline $25 \mathrm{Mn}$ & $5.0 \mathrm{E}-4$ & IAEA94 & $\mathrm{Ng} 82 \mathrm{a}$ & 66 Dy & $2.0 \mathrm{E}-5$ & Based on $\mathrm{Ce}$ & CEC87 \\
\hline $26 \mathrm{Fe}$ & $2.0 \mathrm{E}-2$ & IAEA94 & $\mathrm{Ng} 82 \mathrm{a}$ & $67 \mathrm{Ho}$ & $2.0 \mathrm{E}-5$ & Based on $\mathrm{Ce}$ & CEC87 \\
\hline $27 \mathrm{Co}$ & $1.0 \mathrm{E}-2$ & IAEA94 & $\mathrm{Ng} 82 \mathrm{a}$ & $68 \mathrm{Er}$ & $2.0 \mathrm{E}-5$ & Based on $\mathrm{Ce}$ & CEC87 \\
\hline $28 \mathrm{Ni}$ & $5.0 \mathrm{E}-3$ & IAEA94 & $\mathrm{Cr} 90$ & $72 \mathrm{Hf}$ & $1.0 \mathrm{E}-3$ & $\mathrm{KS} 92$ & Ng68 \\
\hline $29 \mathrm{Cu}$ & $9.0 \mathrm{E}-3$ & IAEA94 & $\mathrm{Ng} 82 \mathrm{a}$ & $73 \mathrm{Ta}$ & $3.0 \mathrm{E}-7$ & Based on $\mathrm{Nb}$ & Jo88 \\
\hline $30 \mathrm{Zn}$ & $1.0 \mathrm{E}-1$ & IAEA94 & $\mathrm{Ng} 82 \mathrm{a}$ & $74 \mathrm{~W}$ & $4.0 \mathrm{E}-2$ & IAEA94 & $\mathrm{Ng} 82 \mathrm{a}$ \\
\hline $31 \mathrm{Ga}$ & $5.0 \mathrm{E}-4$ & $\mathrm{KS} 92$ & $\mathrm{Ba} 84$ & $75 \mathrm{Re}$ & $8.0 \mathrm{E}-3$ & KS92 & Ng68 \\
\hline $33 \mathrm{As}$ & $2.0 \mathrm{E}-3$ & $\mathrm{KS} 92$ & $\mathrm{Ng} 68$ & $76 \mathrm{Os}$ & $4.0 \mathrm{E}-1$ & $\mathrm{KS} 92$ & Ng68 \\
\hline $34 \mathrm{Se}$ & $1.5 \mathrm{E}-2$ & $\mathrm{KS} 92$ & Ng68 & $77 \mathrm{Ir}$ & $1.5 \mathrm{E}-3$ & KS92 & Ng68 \\
\hline $35 \mathrm{Br}$ & $2.5 \mathrm{E}-2$ & $\mathrm{KS} 92$ & Ng68 & $79 \mathrm{Au}$ & $5.0 \mathrm{E}-3$ & $\mathrm{Na} 88$ & NCRP86 \\
\hline $37 \mathrm{Rb}$ & $1.0 \mathrm{E}-2$ & IAEA94 & $\mathrm{Ng} 82 \mathrm{a}$ & $80 \mathrm{Hg}$ & $2.5 \mathrm{E}-1$ & KS92 & Ng68 \\
\hline $38 \mathrm{Sr}$ & $8.0 \mathrm{E}-3$ & IAEA94 & Co90 & $81 \mathrm{Tl}$ & $4.0 \mathrm{E}-2$ & $\mathrm{KS} 92$ & Ng68 \\
\hline $39 \mathrm{Y}$ & $1.0 \mathrm{E}-3$ & IAEA94 & $\mathrm{Ng} 82 \mathrm{a}$ & $82 \mathrm{~Pb}$ & $4.0 \mathrm{E}-4$ & IAEA94 & $\mathrm{Ng} 82 \mathrm{a}$ \\
\hline $40 \mathrm{Zr}$ & $1.0 \mathrm{E}-6$ & IAEA94 & Jo88 & $83 \mathrm{Bi}$ & $4.0 \mathrm{E}-4$ & KS92 & $\mathrm{Ng} 68$ \\
\hline $41 \mathrm{Nb}$ & $3.0 \mathrm{E}-7$ & IAEA94 & Jo88 & $84 \mathrm{Po}$ & $5.0 \mathrm{E}-3$ & IAEA94 & $\mathrm{Ng} 82 \mathrm{a}$ \\
\hline $42 \mathrm{Mo}$ & $1.0 \mathrm{E}-3$ & IAEA94 & Jo88 & $88 \mathrm{Ra}$ & $9.0 \mathrm{E}-4$ & IAEA94 & MB90 \\
\hline $43 \mathrm{Tc}$ & $1.0 \mathrm{E}-4$ & IAEA94 & Bi89 & $89 \mathrm{Ac}$ & $4.0 \mathrm{E}-4$ & $\mathrm{Na} 88$ & LS85 \\
\hline $44 \mathrm{Ru}$ & $5.0 \mathrm{E}-2$ & IAEA94 & $\mathrm{Co} 90$ & $90 \mathrm{Th}$ & $4.0 \mathrm{E}-5$ & Based on Am & $\operatorname{Co} 90$ \\
\hline $45 \mathrm{Rh}$ & $2.0 \mathrm{E}-3$ & KS92 & Ng79 & $91 \mathrm{~Pa}$ & $4.0 \mathrm{E}-5$ & Based on Am & Co90 \\
\hline $46 \mathrm{Pd}$ & $4.0 \mathrm{E}-3$ & $\mathrm{KS} 92$ & Ng68 & $92 \mathrm{U}$ & $3.0 \mathrm{E}-4$ & IAEA94 & $\mathrm{Cr} 90$ \\
\hline $47 \mathrm{Ag}$ & $3.0 \mathrm{E}-3$ & IAEA94 & CEC87 & $93 \mathrm{~Np}$ & $1.0 \mathrm{E}-3$ & IAEA94 & Br79 \\
\hline $48 \mathrm{Cd}$ & $4.0 \mathrm{E}-4$ & IAEA94 & $\mathrm{Ng} 82 \mathrm{a}$ & $94 \mathrm{Pu}$ & $1.0 \mathrm{E}-5$ & IAEA94 & Co90 \\
\hline $49 \mathrm{In}$ & $8.0 \mathrm{E}-3$ & KS92 & Ng68 & $95 \mathrm{Am}$ & $4.0 \mathrm{E}-5$ & IAEA94 & Co90 \\
\hline $50 \mathrm{Sn}$ & $8.0 \mathrm{E}-2$ & KS92 & Ng68 & $96 \mathrm{Cm}$ & $4.0 \mathrm{E}-5$ & Based on Am & Co90 \\
\hline $51 \mathrm{Sb}$ & $1.0 \mathrm{E}-3$ & $\mathrm{Na} 88$ & IAEA 87 & $98 \mathrm{Cf}$ & $4.0 \mathrm{E}-5$ & Based on Am & Co90 \\
\hline
\end{tabular}


Table 2.7. Transfer Factors for Poultry (Bq/kg poultry muscle per $\mathrm{Bq} / \mathrm{d}$ intake)

\begin{tabular}{|c|c|c|c|c|c|c|c|}
\hline $\begin{array}{c}\mathrm{Z} \text { and } \\
\text { Element }\end{array}$ & $\begin{array}{c}\text { Recommended } \\
\text { Value (Wet } \\
\text { Weight) }\end{array}$ & Compiled in & $\begin{array}{c}\text { Primary } \\
\text { Reference }\end{array}$ & $\begin{array}{c}\mathrm{Z} \text { and } \\
\text { Element }\end{array}$ & $\begin{array}{c}\text { Recommended } \\
\text { Value (Wet } \\
\text { Weight) }\end{array}$ & Compiled in & $\begin{array}{c}\text { Primary } \\
\text { Reference }\end{array}$ \\
\hline $04 \mathrm{Be}$ & $4.0 \mathrm{E}-1$ & KS92 & $\mathrm{Na} 88$ & $52 \mathrm{Te}$ & $6.0 \mathrm{E}-1$ & IAEA94 & En88a \\
\hline $07 \mathrm{~N}$ & $9.8 \mathrm{E}-2$ & g.m. & g.m. & $53 \mathrm{I}$ & $5.0 \mathrm{E}-2$ & Sn94 & Sn94 \\
\hline $09 \mathrm{~F}$ & $1.4 \mathrm{E}-2$ & g.m. & g.m. & $55 \mathrm{Cs}$ & $3.0 \mathrm{E}+0$ & Vo93 & Vo93 \\
\hline $11 \mathrm{Na}$ & $1.0 \mathrm{E}-2$ & KS92 & $\mathrm{Na} 88$ & $56 \mathrm{Ba}$ & $9.0 \mathrm{E}-3$ & IAEA94 & En88a \\
\hline $12 \mathrm{Mg}$ & $3.0 \mathrm{E}-2$ & KS92 & KS92 & $57 \mathrm{La}$ & $1.0 \mathrm{E}-1$ & IAEA94 & $\mathrm{Ng} 82 \mathrm{a}$ \\
\hline $14 \mathrm{Si}$ & $8.0 \mathrm{E}-1$ & Based on As & $\mathrm{Na} 88$ & $58 \mathrm{Ce}$ & $2.0 \mathrm{E}-3$ & IAEA94 & $\mathrm{Ng} 82 \mathrm{a}$ \\
\hline $15 \mathrm{P}$ & $1.9 \mathrm{E}-1$ & KS92 & $\mathrm{Na} 88$ & $59 \mathrm{Pr}$ & $2.0 \mathrm{E}-3$ & Based on $\mathrm{Ce}$ & $\mathrm{Ng} 82 \mathrm{a}$ \\
\hline $16 \mathrm{~S}$ & $2.3 \mathrm{E}+0$ & g.m. & g.m. & $60 \mathrm{Nd}$ & $2.0 \mathrm{E}-3$ & Based on $\mathrm{Ce}$ & $\mathrm{Ng} 82 \mathrm{a}$ \\
\hline $17 \mathrm{Cl}$ & $3.0 \mathrm{E}-2$ & KS92 & $\mathrm{Na} 88$ & $61 \mathrm{Pm}$ & $2.0 \mathrm{E}-3$ & Based on $\mathrm{Ce}$ & $\mathrm{Ng} 82 \mathrm{a}$ \\
\hline $19 \mathrm{~K}$ & $4.0 \mathrm{E}-1$ & KS92 & KS92 & $62 \mathrm{Sm}$ & $2.0 \mathrm{E}-3$ & Based on $\mathrm{Ce}$ & $\mathrm{Ng} 82 \mathrm{a}$ \\
\hline $20 \mathrm{Ca}$ & $4.0 \mathrm{E}-2$ & IAEA94 & $\mathrm{Ng} 82 \mathrm{a}$ & $63 \mathrm{Eu}$ & $2.0 \mathrm{E}-3$ & Based on $\mathrm{Ce}$ & $\mathrm{Ng} 82 \mathrm{a}$ \\
\hline $21 \mathrm{Sc}$ & $4.0 \mathrm{E}-3$ & KS92 & $\mathrm{Na} 88$ & $64 \mathrm{Gd}$ & $2.0 \mathrm{E}-3$ & Based on $\mathrm{Ce}$ & $\mathrm{Ng} 82 \mathrm{a}$ \\
\hline $24 \mathrm{Cr}$ & $2.0 \mathrm{E}-1$ & KS92 & KS92 & $65 \mathrm{~Tb}$ & $2.0 \mathrm{E}-3$ & Based on $\mathrm{Ce}$ & $\mathrm{Ng} 82 \mathrm{a}$ \\
\hline $25 \mathrm{Mn}$ & $5.0 \mathrm{E}-2$ & IAEA94 & $\mathrm{Ng} 82 \mathrm{a}$ & 66 Dy & $2.0 \mathrm{E}-3$ & Based on $\mathrm{Ce}$ & $\mathrm{Ng} 82 \mathrm{a}$ \\
\hline $26 \mathrm{Fe}$ & $1.0 \mathrm{E}+0$ & IAEA94 & $\mathrm{Ng} 82 \mathrm{a}$ & $67 \mathrm{Ho}$ & $2.0 \mathrm{E}-3$ & Based on $\mathrm{Ce}$ & $\mathrm{Ng} 82 \mathrm{a}$ \\
\hline $27 \mathrm{Co}$ & $2.0 \mathrm{E}+0$ & IAEA94 & $\mathrm{Ng} 82 \mathrm{a}$ & $68 \mathrm{Er}$ & $2.0 \mathrm{E}-3$ & Based on $\mathrm{Ce}$ & $\mathrm{Ng} 82 \mathrm{a}$ \\
\hline $28 \mathrm{Ni}$ & $1.0 \mathrm{E}-3$ & KS92 & $\mathrm{Na} 88$ & $72 \mathrm{Hf}$ & $6.0 \mathrm{E}-5$ & based on $\mathrm{Zr}$ & En88a \\
\hline $29 \mathrm{Cu}$ & $5.0 \mathrm{E}-1$ & IAEA94 & $\mathrm{Ng} 82 \mathrm{a}$ & $73 \mathrm{Ta}$ & $3.0 \mathrm{E}-4$ & Based on $\mathrm{Nb}$ & En88a \\
\hline $30 \mathrm{Zn}$ & $7.0 \mathrm{E}+0$ & IAEA94 & $\mathrm{Ng} 82 \mathrm{a}$ & $74 \mathrm{~W}$ & $2.0 \mathrm{E}-1$ & Based on Mo & IAEA94 \\
\hline $31 \mathrm{Ga}$ & $8.0 \mathrm{E}-1$ & Based on As & $\mathrm{Na} 88$ & $75 \mathrm{Re}$ & $4.0 \mathrm{E}-2$ & KS92 & $\mathrm{Ba} 84$ \\
\hline $33 \mathrm{As}$ & $8.3 \mathrm{E}-1$ & KS92 & $\mathrm{Na} 88$ & $76 \mathrm{Os}$ & $8.4 \mathrm{E}-2$ & g.m. & g.m. \\
\hline $34 \mathrm{Se}$ & $9.0 \mathrm{E}+0$ & IAEA94 & $\mathrm{Ng} 82 \mathrm{a}$ & $77 \mathrm{Ir}$ & $2.0 \mathrm{E}+0$ & Based on Co & $\mathrm{Ng} 82 \mathrm{a}$ \\
\hline $35 \mathrm{Br}$ & $4.0 \mathrm{E}-3$ & KS92 & $\mathrm{Na} 88$ & $79 \mathrm{Au}$ & $1.0 \mathrm{E}+0$ & g.m. & g.m. \\
\hline $37 \mathrm{Rb}$ & $2.0 \mathrm{E}+0$ & KS92 & $\mathrm{Na} 88$ & $80 \mathrm{Hg}$ & $3.0 \mathrm{E}-2$ & IAEA94 & $\mathrm{Ng} 82 \mathrm{a}$ \\
\hline $38 \mathrm{Sr}$ & $8.0 \mathrm{E}-2$ & IAEA94 & Co90 & $81 \mathrm{Tl}$ & $8.0 \mathrm{E}-1$ & Based on In & $\mathrm{Na} 88$ \\
\hline $39 \mathrm{Y}$ & $1.0 \mathrm{E}-2$ & IAEA94 & $\mathrm{Ng} 82 \mathrm{a}$ & $82 \mathrm{~Pb}$ & $8.0 \mathrm{E}-1$ & Based on As & $\mathrm{Na} 88$ \\
\hline $40 \mathrm{Zr}$ & $6.0 \mathrm{E}-5$ & IAEA94 & En88a & $83 \mathrm{Bi}$ & $9.8 \mathrm{E}-2$ & g.m. & g.m. \\
\hline $41 \mathrm{Nb}$ & $3.0 \mathrm{E}-4$ & IAEA94 & En88a & $84 \mathrm{Po}$ & $2.3 \mathrm{E}+0$ & g.m. & g.m. \\
\hline $42 \mathrm{Mo}$ & $1.8 \mathrm{E}-1$ & En88a & En88a & $88 \mathrm{Ra}$ & $3.0 \mathrm{E}-2$ & KS92 & KS92 \\
\hline $43 \mathrm{Tc}$ & $3.0 \mathrm{E}-2$ & IAEA94 & En88b & $89 \mathrm{Ac}$ & $6.0 \mathrm{E}-3$ & Based on Am & Co90 \\
\hline $44 \mathrm{Ru}$ & $7.0 \mathrm{E}-3$ & $\mathrm{Na} 88$ & $\mathrm{Ng} 82 \mathrm{a}$ & $90 \mathrm{Th}$ & $6.0 \mathrm{E}-3$ & Based on Am & Co90 \\
\hline $45 \mathrm{Rh}$ & $2.0 \mathrm{E}+0$ & Based on Co & $\mathrm{Ng} 82 \mathrm{a}$ & $91 \mathrm{~Pa}$ & $6.0 \mathrm{E}-3$ & Based on Am & Co90 \\
\hline $46 \mathrm{Pd}$ & $3.0 \mathrm{E}-4$ & KS92 & $\mathrm{Na} 88$ & $92 \mathrm{U}$ & $1.0 \mathrm{E}+0$ & IAEA94 & $\mathrm{Ng} 82 \mathrm{a}$ \\
\hline $47 \mathrm{Ag}$ & $2.0 \mathrm{E}+0$ & IAEA94 & CEC87 & $93 \mathrm{~Np}$ & $6.0 \mathrm{E}-3$ & Based on Am & Co90 \\
\hline $48 \mathrm{Cd}$ & $8.0 \mathrm{E}-1$ & IAEA94 & $\mathrm{Ng} 82 \mathrm{a}$ & $94 \mathrm{Pu}$ & $3.0 \mathrm{E}-3$ & IAEA94 & Co90 \\
\hline $49 \mathrm{In}$ & $8.0 \mathrm{E}-1$ & Based on As & $\mathrm{Na} 88$ & $95 \mathrm{Am}$ & $6.0 \mathrm{E}-3$ & IAEA94 & Co90 \\
\hline $50 \mathrm{Sn}$ & $8.0 \mathrm{E}-1$ & Based on As & $\mathrm{Na} 88$ & $96 \mathrm{Cm}$ & $6.0 \mathrm{E}-3$ & Based on Am & $\mathrm{Co} 90$ \\
\hline $51 \mathrm{Sb}$ & $6.0 \mathrm{E}-3$ & $\mathrm{Na} 88$ & $\mathrm{Na} 88$ & $98 \mathrm{Cf}$ & $6.0 \mathrm{E}-3$ & Based on Am & Co90 \\
\hline
\end{tabular}


Table 2.8. Transfer Factors for Milk ( $\mathrm{Bq} / \mathrm{L}$ milk per $\mathrm{Bq} / \mathrm{d}$ intake)

\begin{tabular}{|c|c|c|c|c|c|c|c|}
\hline $\begin{array}{c}\mathrm{Z} \text { and } \\
\text { Element }\end{array}$ & $\begin{array}{c}\text { Recommended } \\
\text { Value (Wet } \\
\text { Weight) }\end{array}$ & Compiled in & $\begin{array}{c}\text { Primary } \\
\text { Reference }\end{array}$ & $\begin{array}{c}\mathrm{Z} \text { and } \\
\text { Element }\end{array}$ & $\begin{array}{l}\text { Recommended } \\
\text { Value (Wet } \\
\text { Weight) }\end{array}$ & Compiled in & $\begin{array}{c}\text { Primary } \\
\text { Reference }\end{array}$ \\
\hline $01 \mathrm{H}$ & $1.5 \mathrm{E}-2$ & IAEA94 & Va83 & $51 \mathrm{Sb}$ & $2.5 \mathrm{E}-5$ & IAEA94 & Va82 \\
\hline $04 \mathrm{Be}$ & $9.0 \mathrm{E}-7$ & KS92 & $\mathrm{Ng} 77$ & $52 \mathrm{Te}$ & 4.5E-4 & IAEA94 & Jo88 \\
\hline $06 \mathrm{C}$ & ND & & & $53 \mathrm{I}$ & $9.0 \mathrm{E}-3$ & $\operatorname{Sn} 94$ & Sn94 \\
\hline $07 \mathrm{~N}$ & $2.5 \mathrm{E}-2$ & KS92 & $\mathrm{Ng} 77$ & $55 \mathrm{Cs}$ & 7.9E-3 & IAEA94 & Co90 \\
\hline $09 \mathrm{~F}$ & $1.0 \mathrm{E}-3$ & KS92 & $\mathrm{Ng} 77$ & $56 \mathrm{Ba}$ & $4.8 \mathrm{E}-4$ & IAEA94 & Jo88 \\
\hline $11 \mathrm{Na}$ & $1.6 \mathrm{E}-2$ & IAEA94 & IAEA94 & $57 \mathrm{La}$ & $2.0 \mathrm{E}-5$ & KS92 & $\mathrm{Ng} 77$ \\
\hline $12 \mathrm{Mg}$ & $3.9 \mathrm{E}-3$ & IAEA94 & $\mathrm{Ng} 82 \mathrm{a}$ & $58 \mathrm{Ce}$ & $3.0 \mathrm{E}-5$ & IAEA94 & CEC87 \\
\hline $14 \mathrm{Si}$ & $2.0 \mathrm{E}-5$ & KS92 & $\mathrm{Ng} 77$ & $59 \operatorname{Pr}$ & $3.0 \mathrm{E}-5$ & Based on $\mathrm{Ce}$ & CEC87 \\
\hline $15 \mathrm{P}$ & $1.6 \mathrm{E}-2$ & IAEA94 & $\mathrm{Ng} 82 \mathrm{a}$ & $60 \mathrm{Nd}$ & $3.0 \mathrm{E}-5$ & Based on $\mathrm{Ce}$ & CEC87 \\
\hline $16 \mathrm{~S}$ & $1.6 \mathrm{E}-2$ & IAEA94 & $\mathrm{Ng} 82 \mathrm{a}$ & $61 \mathrm{Pm}$ & $3.0 \mathrm{E}-5$ & Based on $\mathrm{Ce}$ & CEC87 \\
\hline $17 \mathrm{Cl}$ & $1.7 \mathrm{E}-2$ & IAEA94 & Bi89 & $62 \mathrm{Sm}$ & $3.0 \mathrm{E}-5$ & Based on $\mathrm{Ce}$ & CEC87 \\
\hline $19 \mathrm{~K}$ & 7.2E-3 & IAEA94 & $\mathrm{Ng} 82 \mathrm{a}$ & $63 \mathrm{Eu}$ & $3.0 \mathrm{E}-5$ & Based on $\mathrm{Ce}$ & CEC87 \\
\hline $20 \mathrm{Ca}$ & $3.0 \mathrm{E}-3$ & IAEA94 & СТ83 & $64 \mathrm{Gd}$ & $3.0 \mathrm{E}-5$ & Based on $\mathrm{Ce}$ & CEC87 \\
\hline $21 \mathrm{Sc}$ & $5.0 \mathrm{E}-6$ & KS92 & $\mathrm{Ng} 77$ & $65 \mathrm{~Tb}$ & $3.0 \mathrm{E}-5$ & Based on $\mathrm{Ce}$ & CEC87 \\
\hline $24 \mathrm{Cr}$ & $1.0 \mathrm{E}-5$ & IAEA94 & Va84 & 66 Dy & $3.0 \mathrm{E}-5$ & Based on $\mathrm{Ce}$ & CEC87 \\
\hline $25 \mathrm{Mn}$ & $3.0 \mathrm{E}-5$ & IAEA94 & Va84 & $67 \mathrm{Ho}$ & $3.0 \mathrm{E}-5$ & Based on $\mathrm{Ce}$ & CEC87 \\
\hline $26 \mathrm{Fe}$ & $3.0 \mathrm{E}-5$ & IAEA94 & Va84 & $68 \mathrm{Er}$ & $3.0 \mathrm{E}-5$ & Based on $\mathrm{Ce}$ & CEC87 \\
\hline $27 \mathrm{Co}$ & $3.0 \mathrm{E}-4$ & IAEA94 & $\mathrm{Ba} 84$ & $72 \mathrm{Hf}$ & $5.5 \mathrm{E}-7$ & Based on $\mathrm{Zr}$ & Jo88 \\
\hline $28 \mathrm{Ni}$ & $1.6 \mathrm{E}-2$ & IAEA94 & $\mathrm{Cr} 90$ & $73 \mathrm{Ta}$ & 4.1E-7 & Based on $\mathrm{Nb}$ & Jo88 \\
\hline $29 \mathrm{Cu}$ & $2.0 \mathrm{E}-3$ & $\mathrm{Na} 88$ & IAEA87 & $74 \mathrm{~W}$ & $3.0 \mathrm{E}-4$ & KS92 & $\mathrm{Ng} 77$ \\
\hline $30 \mathrm{Zn}$ & $1.0 \mathrm{E}-2$ & KS92 & $\mathrm{Ng} 77$ & $75 \mathrm{Re}$ & $1.5 \mathrm{E}-3$ & $\mathrm{KS} 92$ & $\mathrm{Ng} 77$ \\
\hline $31 \mathrm{Ga}$ & $5.0 \mathrm{E}-5$ & KS92 & $\mathrm{Ng} 77$ & $76 \mathrm{Os}$ & $5.0 \mathrm{E}-3$ & KS92 & $\mathrm{Ng} 77$ \\
\hline $33 \mathrm{As}$ & $6.0 \mathrm{E}-5$ & KS92 & $\mathrm{Ng} 77$ & $77 \mathrm{Ir}$ & $2.0 \mathrm{E}-6$ & $\mathrm{KS} 92$ & $\mathrm{Ng} 77$ \\
\hline $34 \mathrm{Se}$ & $4.0 \mathrm{E}-3$ & KS92 & $\mathrm{Ng} 77$ & $79 \mathrm{Au}$ & $5.5 \mathrm{E}-6$ & $\mathrm{KS} 92$ & $\mathrm{Ng} 77$ \\
\hline $35 \mathrm{Br}$ & $2.0 \mathrm{E}-2$ & KS92 & $\mathrm{Ng} 77$ & $80 \mathrm{Hg}$ & 4.7E-4 & IAEA94 & $\mathrm{Ng} 82 \mathrm{a}$ \\
\hline $37 \mathrm{Rb}$ & $1.2 \mathrm{E}-2$ & IAEA94 & $\mathrm{Ng} 82 \mathrm{a}$ & $81 \mathrm{Tl}$ & $2.0 \mathrm{E}-3$ & $\mathrm{Na} 88$ & $\mathrm{Na} 88$ \\
\hline $38 \mathrm{Sr}$ & $2.8 \mathrm{E}-3$ & IAEA94 & Co90 & $82 \mathrm{~Pb}$ & $2.6 \mathrm{E}-4$ & KS92 & $\mathrm{Ng} 77$ \\
\hline $39 \mathrm{Y}$ & $2.0 \mathrm{E}-5$ & KS92 & $\mathrm{Ng} 77$ & $83 \mathrm{Bi}$ & $5.0 \mathrm{E}-4$ & KS92 & $\mathrm{Ng} 77$ \\
\hline $40 \mathrm{Zr}$ & $5.5 \mathrm{E}-7$ & IAEA94 & Jo88 & $84 \mathrm{Po}$ & $3.4 \mathrm{E}-4$ & IAEA94 & $\mathrm{Ng} 82 \mathrm{a}$ \\
\hline $41 \mathrm{Nb}$ & 4.1E-7 & IAEA94 & Jo88 & $88 \mathrm{Ra}$ & $1.3 \mathrm{E}-3$ & IAEA94 & MB90 \\
\hline $42 \mathrm{Mo}$ & $1.7 \mathrm{E}-3$ & IAEA94 & Jo88 & $89 \mathrm{Ac}$ & $2.0 \mathrm{E}-5$ & KS92 & $\mathrm{Ng} 77$ \\
\hline $43 \mathrm{Tc}$ & $1.4 \mathrm{E}-4$ & IAEA94 & Jo88 & $90 \mathrm{Th}$ & $5.0 \mathrm{E}-6$ & $\mathrm{KS} 92$ & $\mathrm{Ng} 77$ \\
\hline $44 \mathrm{Ru}$ & $3.3 \mathrm{E}-6$ & IAEA94 & Co90 & $91 \mathrm{~Pa}$ & $5.0 \mathrm{E}-6$ & $\mathrm{KS} 92$ & $\mathrm{Ng} 77$ \\
\hline $45 \mathrm{Rh}$ & $1.0 \mathrm{E}-2$ & KS92 & $\mathrm{Ng} 77$ & $92 \mathrm{U}$ & $4.0 \mathrm{E}-4$ & IAEA94 & MB90 \\
\hline $46 \mathrm{Pd}$ & $1.0 \mathrm{E}-2$ & $\mathrm{KS} 92$ & $\mathrm{Ng} 77$ & $93 \mathrm{~Np}$ & $5.0 \mathrm{E}-6$ & IAEA94 & $\mathrm{Ng} 82 \mathrm{a}$ \\
\hline $47 \mathrm{Ag}$ & $5.0 \mathrm{E}-5$ & IAEA94 & CEC87 & $94 \mathrm{Pu}$ & $1.1 \mathrm{E}-6$ & IAEA94 & Co90 \\
\hline $48 \mathrm{Cd}$ & $1.0 \mathrm{E}-3$ & KS92 & $\mathrm{Ng} 77$ & $95 \mathrm{Am}$ & $1.5 \mathrm{E}-6$ & IAEA94 & Co90 \\
\hline $49 \mathrm{In}$ & $2.0 \mathrm{E}-4$ & $\mathrm{Na} 88$ & NCRP86 & $96 \mathrm{Cm}$ & $2.0 \mathrm{E}-5$ & KS92 & $\mathrm{Ng} 77$ \\
\hline $50 \mathrm{Sn}$ & $1.0 \mathrm{E}-3$ & $\mathrm{Na} 88$ & NCRP86 & $98 \mathrm{Cf}$ & $1.5 \mathrm{E}-6$ & Based on Am & $\mathrm{Co} 90$ \\
\hline
\end{tabular}


Table 2.9. Transfer Factors for Eggs (Bq/kg egg per Bq/d intake)

\begin{tabular}{|c|c|c|c|c|c|c|c|}
\hline $\begin{array}{c}\mathrm{Z} \text { and } \\
\text { Element }\end{array}$ & $\begin{array}{c}\text { Recommended } \\
\text { Value (Wet } \\
\text { Weight) }\end{array}$ & $\begin{array}{c}\text { Compiled } \\
\text { in }\end{array}$ & $\begin{array}{c}\text { Primary } \\
\text { Reference }\end{array}$ & $\begin{array}{c}\mathrm{Z} \text { and } \\
\text { Element }\end{array}$ & $\begin{array}{c}\text { Recommended } \\
\text { Value (Wet } \\
\text { Weight) } \\
\end{array}$ & $\begin{array}{c}\text { Compiled } \\
\text { in }\end{array}$ & $\begin{array}{c}\text { Primary } \\
\text { Reference }\end{array}$ \\
\hline $04 \mathrm{Be}$ & $2.0 \mathrm{E}-2$ & KS92 & $\mathrm{Na} 88$ & $52 \mathrm{Te}$ & $5.0 \mathrm{E}+0$ & IAEA94 & En88a \\
\hline $07 \mathrm{~N}$ & $2.6 \mathrm{E}-1$ & g.m. & g.m. & $53 \mathrm{I}$ & $4.4 \mathrm{E}+0$ & Sn94 & Sn94 \\
\hline $09 \mathrm{~F}$ & $2.7 \mathrm{E}+0$ & g.m. & g.m. & $55 \mathrm{Cs}$ & $4.0 \mathrm{E}-1$ & IAEA94 & Co90 \\
\hline $11 \mathrm{Na}$ & $6.0 \mathrm{E}+0$ & IAEA94 & $\mathrm{Ng} 82 \mathrm{a}$ & $56 \mathrm{Ba}$ & $9.0 \mathrm{E}-1$ & IAEA94 & En88a \\
\hline $12 \mathrm{Mg}$ & $2.0 \mathrm{E}+0$ & IAEA94 & $\mathrm{Ng} 82 \mathrm{a}$ & $57 \mathrm{La}$ & $9.0 \mathrm{E}-3$ & IAEA94 & $\mathrm{Ng} 82 \mathrm{a}$ \\
\hline $14 \mathrm{Si}$ & $1.0 \mathrm{E}+0$ & Based on $\mathrm{P}$ & $\mathrm{Na} 88$ & $58 \mathrm{Ce}$ & $4.0 \mathrm{E}-5$ & IAEA94 & CEC87 \\
\hline $15 \mathrm{P}$ & $1.0 \mathrm{E}+0$ & KS92 & $\mathrm{Na} 88$ & $59 \operatorname{Pr}$ & $4.0 \mathrm{E}-5$ & Based on $\mathrm{Ce}$ & CEC87 \\
\hline $16 \mathrm{~S}$ & $7.0 \mathrm{E}+0$ & g.m. & g.m. & $60 \mathrm{Nd}$ & $4.0 \mathrm{E}-5$ & Based on $\mathrm{Ce}$ & CEC87 \\
\hline $17 \mathrm{Cl}$ & $2.7 \mathrm{E}+0$ & g.m. & g.m. & $61 \mathrm{Pm}$ & $4.0 \mathrm{E}-5$ & Based on $\mathrm{Ce}$ & CEC87 \\
\hline $19 \mathrm{~K}$ & $1.0 \mathrm{E}+0$ & IAEA94 & $\mathrm{Ng} 82 \mathrm{a}$ & $62 \mathrm{Sm}$ & $4.0 \mathrm{E}-5$ & Based on $\mathrm{Ce}$ & CEC87 \\
\hline $20 \mathrm{Ca}$ & $4.0 \mathrm{E}-1$ & IAEA94 & $\mathrm{Ng} 82 \mathrm{a}$ & $63 \mathrm{Eu}$ & $4.0 \mathrm{E}-5$ & Based on $\mathrm{Ce}$ & CEC87 \\
\hline $21 \mathrm{Sc}$ & $4.2 \mathrm{E}-3$ & g.m. & g.m. & $64 \mathrm{Gd}$ & $4.0 \mathrm{E}-5$ & Based on $\mathrm{Ce}$ & CEC87 \\
\hline $24 \mathrm{Cr}$ & $9.0 \mathrm{E}-1$ & Based on Mo & En88a & $65 \mathrm{~Tb}$ & $4.0 \mathrm{E}-5$ & Based on $\mathrm{Ce}$ & CEC87 \\
\hline $25 \mathrm{Mn}$ & $6.0 \mathrm{E}-2$ & IAEA94 & $\mathrm{Ng} 82 \mathrm{a}$ & 66 Dy & $4.0 \mathrm{E}-5$ & Based on $\mathrm{Ce}$ & CEC87 \\
\hline $26 \mathrm{Fe}$ & $1.0 \mathrm{E}+0$ & IAEA94 & $\mathrm{Ng} 82 \mathrm{a}$ & $67 \mathrm{Ho}$ & $4.0 \mathrm{E}-5$ & Based on $\mathrm{Ce}$ & CEC87 \\
\hline $27 \mathrm{Co}$ & $1.0 \mathrm{E}-1$ & IAEA94 & $\mathrm{Ng} 82 \mathrm{a}$ & $68 \mathrm{Er}$ & $4.0 \mathrm{E}-5$ & Based on $\mathrm{Ce}$ & CEC87 \\
\hline $28 \mathrm{Ni}$ & $1.0 \mathrm{E}-1$ & KS92 & $\mathrm{Na} 88$ & $72 \mathrm{Hf}$ & $2.0 \mathrm{E}-4$ & Based on $\mathrm{Zr}$ & Jo88 \\
\hline $29 \mathrm{Cu}$ & $5.0 \mathrm{E}-1$ & IAEA94 & $\mathrm{Ng} 82 \mathrm{a}$ & $73 \mathrm{Ta}$ & $1.0 \mathrm{E}-3$ & Based on $\mathrm{Nb}$ & En88a \\
\hline $30 \mathrm{Zn}$ & $3.0 \mathrm{E}+0$ & IAEA94 & $\mathrm{Ng} 82 \mathrm{a}$ & $74 \mathrm{~W}$ & $9.0 \mathrm{E}-1$ & Based on Mo & En88a \\
\hline $31 \mathrm{Ga}$ & $1.0 \mathrm{E}+0$ & Based on $\mathrm{P}$ & $\mathrm{Na} 88$ & $75 \mathrm{Re}$ & $4.2 \mathrm{E}-1$ & g.m. & g.m. \\
\hline $33 \mathrm{As}$ & $2.6 \mathrm{E}-1$ & g.m. & g.m. & $76 \mathrm{Os}$ & 7.1E-2 & g.m. & g.m. \\
\hline $34 \mathrm{Se}$ & $9.0 \mathrm{E}+0$ & IAEA94 & $\mathrm{Ng} 82 \mathrm{a}$ & $77 \mathrm{Ir}$ & $1.0 \mathrm{E}-1$ & Based on Co & $\mathrm{Ng} 82 \mathrm{a}$ \\
\hline $35 \mathrm{Br}$ & $1.6 \mathrm{E}+0$ & $\mathrm{KS} 92$ & $\mathrm{Na} 88$ & $79 \mathrm{Au}$ & $5.0 \mathrm{E}-1$ & Based on $\mathrm{Cu}$ & $\mathrm{Ng} 82 \mathrm{a}$ \\
\hline $37 \mathrm{Rb}$ & $3.0 \mathrm{E}+0$ & KS92 & $\mathrm{Na} 88$ & $80 \mathrm{Hg}$ & $5.0 \mathrm{E}-1$ & g.m. & g.m. \\
\hline $38 \mathrm{Sr}$ & $2.0 \mathrm{E}-1$ & IAEA94 & Co90 & $81 \mathrm{Tl}$ & $1.0 \mathrm{E}+0$ & Based on $\mathrm{P}$ & $\mathrm{Na} 88$ \\
\hline $39 \mathrm{Y}$ & $2.0 \mathrm{E}-3$ & IAEA94 & $\mathrm{Ng} 82 \mathrm{a}$ & $82 \mathrm{~Pb}$ & $1.0 \mathrm{E}+0$ & Based on P & $\mathrm{Na} 88$ \\
\hline $40 \mathrm{Zr}$ & $2.0 \mathrm{E}-4$ & IAEA94 & En88a & $83 \mathrm{Bi}$ & $2.6 \mathrm{E}-1$ & g.m. & g.m. \\
\hline $41 \mathrm{Nb}$ & $1.0 \mathrm{E}-3$ & IAEA94 & En88a & $84 \mathrm{Po}$ & $7.0 \mathrm{E}+0$ & g.m. & g.m. \\
\hline $42 \mathrm{Mo}$ & $9.0 \mathrm{E}-1$ & IAEA94 & En88a & $88 \mathrm{Ra}$ & $3.1 \mathrm{E}-1$ & g.m. & g.m. \\
\hline $43 \mathrm{Tc}$ & $3.0 \mathrm{E}+0$ & IAEA94 & En88a & $89 \mathrm{Ac}$ & $4.0 \mathrm{E}-3$ & Based on Am & $\mathrm{Ng} 82 \mathrm{a}$ \\
\hline $44 \mathrm{Ru}$ & $5.0 \mathrm{E}-3$ & IAEA94 & $\mathrm{Co} 90$ & $90 \mathrm{Th}$ & $4.0 \mathrm{E}-3$ & Based on Am & $\mathrm{Ng} 82 \mathrm{a}$ \\
\hline $45 \mathrm{Rh}$ & $1.0 \mathrm{E}-1$ & Based on Co & $\mathrm{Ng} 82 \mathrm{a}$ & $91 \mathrm{~Pa}$ & $4.0 \mathrm{E}-3$ & Based on Am & $\mathrm{Ng} 82 \mathrm{a}$ \\
\hline $46 \mathrm{Pd}$ & $4.0 \mathrm{E}-3$ & $\mathrm{KS} 92$ & $\mathrm{Na} 88$ & $92 \mathrm{U}$ & $1.0 \mathrm{E}+0$ & IAEA94 & $\mathrm{Ng} 82 \mathrm{a}$ \\
\hline $47 \mathrm{Ag}$ & $5.0 \mathrm{E}-1$ & Based on $\mathrm{Cu}$ & $\mathrm{Ng} 82 \mathrm{a}$ & $93 \mathrm{~Np}$ & $4.0 \mathrm{E}-3$ & Based on Am & $\mathrm{Ng} 82 \mathrm{a}$ \\
\hline $48 \mathrm{Cd}$ & $1.0 \mathrm{E}-1$ & IAEA94 & $\mathrm{Ng} 82 \mathrm{a}$ & $94 \mathrm{Pu}$ & $5.0 \mathrm{E}-4$ & IAEA94 & $\mathrm{Ng} 82 \mathrm{a}$ \\
\hline 49 In & $1.0 \mathrm{E}+0$ & Based on $\mathrm{P}$ & $\mathrm{Na} 88$ & $95 \mathrm{Am}$ & $4.0 \mathrm{E}-3$ & IAEA94 & $\mathrm{Ng} 82 \mathrm{a}$ \\
\hline $50 \mathrm{Sn}$ & $1.0 \mathrm{E}+0$ & Based on $\mathrm{P}$ & $\mathrm{Na} 88$ & $96 \mathrm{Cm}$ & $4.0 \mathrm{E}-3$ & Based on Am & $\mathrm{Ng} 82 \mathrm{a}$ \\
\hline $51 \mathrm{Sb}$ & $7.0 \mathrm{E}-2$ & KS92 & $\mathrm{Na} 88$ & $98 \mathrm{Cf}$ & $4.0 \mathrm{E}-3$ & Based on Am & $\mathrm{Ng} 82 \mathrm{a}$ \\
\hline
\end{tabular}

g.m. $=$ geometric mean. 
Table 2.10. Concentration Ratios for Freshwater Fish $(\mathrm{Bq} / \mathrm{kg}$ muscle per $\mathrm{Bq} / \mathrm{L})$

\begin{tabular}{|c|c|c|c|c|c|c|c|}
\hline $\begin{array}{c}\mathrm{Z} \text { and } \\
\text { Element }\end{array}$ & $\begin{array}{c}\text { Recommended } \\
\text { Value (Wet } \\
\text { Weight) }\end{array}$ & $\begin{array}{c}\text { Compiled } \\
\text { in }\end{array}$ & $\begin{array}{c}\text { Primary } \\
\text { Reference }\end{array}$ & $\begin{array}{c}\mathrm{Z} \text { and } \\
\text { Element }\end{array}$ & $\begin{array}{c}\text { Recommended } \\
\text { Value (Wet } \\
\text { Weight) }\end{array}$ & $\begin{array}{c}\text { Compiled } \\
\text { in }\end{array}$ & $\begin{array}{c}\text { Primary } \\
\text { Reference }\end{array}$ \\
\hline $02 \mathrm{He}$ & $1.0 \mathrm{E}+0$ & IAEA94 & IAEA94 & $51 \mathrm{Sb}$ & $1.0 \mathrm{E}+2$ & IAEA94 & IAEA82 \\
\hline $04 \mathrm{Be}$ & $1.0 \mathrm{E}+2$ & IAEA94 & IAEA94 & $52 \mathrm{Te}$ & $4.0 \mathrm{E}+2$ & IAEA94 & IAEA82 \\
\hline $06 \mathrm{C}$ & $5.0 \mathrm{E}+4$ & IAEA94 & IAEA94 & $53 \mathrm{I}$ & $4.0 \mathrm{E}+1$ & IAEA94 & IAEA82 \\
\hline $07 \mathrm{~N}$ & $2.0 \mathrm{E}+5$ & IAEA94 & IAEA94 & $55 \mathrm{Cs}$ & $2.0 \mathrm{E}+3$ & IAEA94 & IAEA82 \\
\hline $08 \mathrm{O}$ & $1.0 \mathrm{E}+0$ & IAEA94 & IAEA94 & $56 \mathrm{Ba}$ & $4.0 \mathrm{E}+0$ & IAEA94 & IAEA82 \\
\hline $09 \mathrm{~F}$ & $1.0 \mathrm{E}+1$ & KS92 & St86 & $57 \mathrm{La}$ & $3.0 \mathrm{E}+1$ & IAEA94 & IAEA82 \\
\hline $11 \mathrm{Na}$ & $2.0 \mathrm{E}+1$ & IAEA94 & IAEA82 & $58 \mathrm{Ce}$ & $3.0 \mathrm{E}+1$ & IAEA94 & IAEA82 \\
\hline $12 \mathrm{Mg}$ & $5.0 \mathrm{E}+1$ & NCRP96 & NCRP96 & $59 \mathrm{Pr}$ & $3.0 \mathrm{E}+1$ & Based on $\mathrm{Ce}$ & IAEA 82 \\
\hline $14 \mathrm{Si}$ & $2.0 \mathrm{E}+1$ & NCRP96 & NCRP96 & $60 \mathrm{Nd}$ & $3.0 \mathrm{E}+1$ & Based on $\mathrm{Ce}$ & IAEA 82 \\
\hline $15 \mathrm{P}$ & $5.0 \mathrm{E}+4$ & IAEA94 & IAEA82 & $61 \mathrm{Pm}$ & $3.0 \mathrm{E}+1$ & Based on $\mathrm{Ce}$ & IAEA82 \\
\hline $16 \mathrm{~S}$ & $8.0 \mathrm{E}+2$ & IAEA94 & IAEA82 & $62 \mathrm{Sm}$ & $3.0 \mathrm{E}+1$ & Based on $\mathrm{Ce}$ & IAEA82 \\
\hline $17 \mathrm{Cl}$ & $5.0 \mathrm{E}+1$ & KS92 & St86 & $63 \mathrm{Eu}$ & $3.0 \mathrm{E}+1$ & Based on $\mathrm{Ce}$ & IAEA82 \\
\hline $19 \mathrm{~K}$ & $1.0 \mathrm{E}+3$ & KS92 & St86 & $64 \mathrm{Gd}$ & $3.0 \mathrm{E}+1$ & Based on $\mathrm{Ce}$ & IAEA82 \\
\hline $20 \mathrm{Ca}$ & $4.0 \mathrm{E}+1$ & KS92 & St86 & $65 \mathrm{~Tb}$ & $3.0 \mathrm{E}+1$ & Based on $\mathrm{Ce}$ & IAEA82 \\
\hline $21 \mathrm{Sc}$ & $1.0 \mathrm{E}+2$ & IAEA94 & IAEA94 & 66 Dy & $3.0 \mathrm{E}+1$ & Based on $\mathrm{Ce}$ & IAEA82 \\
\hline $24 \mathrm{Cr}$ & $4.0 \mathrm{E}+0$ & $\mathrm{Sn} 94$ & Th94 & $67 \mathrm{Ho}$ & $3.0 \mathrm{E}+1$ & Based on $\mathrm{Ce}$ & IAEA 82 \\
\hline $25 \mathrm{Mn}$ & $4.0 \mathrm{E}+2$ & IAEA94 & IAEA82 & $68 \mathrm{Er}$ & $3.0 \mathrm{E}+1$ & Based on $\mathrm{Ce}$ & IAEA 82 \\
\hline $26 \mathrm{Fe}$ & $2.0 \mathrm{E}+2$ & IAEA94 & IAEA82 & $72 \mathrm{Hf}$ & $3.0 \mathrm{E}+2$ & Based on $\mathrm{Zr}$ & IAEA82 \\
\hline $27 \mathrm{Co}$ & $3.0 \mathrm{E}+2$ & IAEA94 & IAEA82 & $73 \mathrm{Ta}$ & $3.0 \mathrm{E}+2$ & Based on $\mathrm{Nb}$ & IAEA94 \\
\hline $28 \mathrm{Ni}$ & $1.0 \mathrm{E}+2$ & IAEA94 & IAEA82 & $74 \mathrm{~W}$ & $1.0 \mathrm{E}+1$ & Based on Mo & IAEA94 \\
\hline $29 \mathrm{Cu}$ & $2.0 \mathrm{E}+2$ & IAEA94 & IAEA94 & $75 \mathrm{Re}$ & $1.2 \mathrm{E}+2$ & $\mathrm{KS} 92$ & St86 \\
\hline $30 \mathrm{Zn}$ & $3.5 \mathrm{E}+2$ & Sn94 & Th94 & $76 \mathrm{Os}$ & $1.0 \mathrm{E}+1$ & KS92 & St86 \\
\hline $31 \mathrm{Ga}$ & $4.0 \mathrm{E}+2$ & NCRP96 & NCRP96 & $77 \mathrm{Ir}$ & $1.0 \mathrm{E}+1$ & $\mathrm{KS} 92$ & St86 \\
\hline $33 \mathrm{As}$ & $1.7 \mathrm{E}+3$ & Sn94 & Th94 & $79 \mathrm{Au}$ & $3.3 \mathrm{E}+1$ & KS92 & St86 \\
\hline $34 \mathrm{Se}$ & $1.7 \mathrm{E}+2$ & KS92 & St86 & $80 \mathrm{Hg}$ & $1.0 \mathrm{E}+3$ & KS92 & St86 \\
\hline $35 \mathrm{Br}$ & $4.0 \mathrm{E}+2$ & IAEA94 & IAEA94 & $81 \mathrm{Tl}$ & $1.0 \mathrm{E}+4$ & NCRP96 & NCRP96 \\
\hline $37 \mathrm{Rb}$ & $2.0 \mathrm{E}+3$ & IAEA94 & IAEA94 & $82 \mathrm{~Pb}$ & $3.0 \mathrm{E}+2$ & IAEA94 & IAEA82 \\
\hline $38 \mathrm{Sr}$ & $6.0 \mathrm{E}+1$ & IAEA94 & IAEA82 & $83 \mathrm{Bi}$ & $1.5 \mathrm{E}+1$ & KS92 & St86 \\
\hline $39 \mathrm{Y}$ & $3.0 \mathrm{E}+1$ & IAEA94 & IAEA82 & $84 \mathrm{Po}$ & $5.0 \mathrm{E}+1$ & IAEA94 & IAEA 82 \\
\hline $40 \mathrm{Zr}$ & $3.0 \mathrm{E}+2$ & IAEA94 & IAEA82 & $88 \mathrm{Ra}$ & $5.0 \mathrm{E}+1$ & IAEA94 & IAEA82 \\
\hline $41 \mathrm{Nb}$ & $3.0 \mathrm{E}+2$ & IAEA94 & IAEA82 & $89 \mathrm{Ac}$ & $2.5 \mathrm{E}+1$ & KS92 & St86 \\
\hline $42 \mathrm{Mo}$ & $1.0 \mathrm{E}+1$ & IAEA94 & IAEA94 & $90 \mathrm{Th}$ & $1.0 \mathrm{E}+2$ & KS92 & $\mathrm{Na} 88$ \\
\hline $43 \mathrm{Tc}$ & $2.0 \mathrm{E}+1$ & IAEA94 & IAEA82 & $91 \mathrm{~Pa}$ & $1.0 \mathrm{E}+1$ & NCRP96 & NCRP96 \\
\hline $44 \mathrm{Ru}$ & $1.0 \mathrm{E}+2$ & KS92 & $\mathrm{Na} 88$ & $92 \mathrm{U}$ & $1.0 \mathrm{E}+1$ & IAEA94 & IAEA82 \\
\hline $45 \mathrm{Rh}$ & $1.0 \mathrm{E}+1$ & IAEA94 & IAEA94 & $93 \mathrm{~Np}$ & $2.1 \mathrm{E}+1$ & Sn94 & Th94 \\
\hline $46 \mathrm{Pd}$ & $1.0 \mathrm{E}+1$ & KS92 & St86 & $94 \mathrm{Pu}$ & $3.0 \mathrm{E}+1$ & IAEA94 & IAEA 82 \\
\hline $47 \mathrm{Ag}$ & $5.0 \mathrm{E}+0$ & IAEA94 & IAEA82 & $95 \mathrm{Am}$ & $3.0 \mathrm{E}+1$ & IAEA94 & IAEA82 \\
\hline $48 \mathrm{Cd}$ & $2.0 \mathrm{E}+2$ & $\mathrm{KS} 92$ & St86 & $96 \mathrm{Cm}$ & $3.0 \mathrm{E}+1$ & IAEA94 & IAEA 82 \\
\hline 49 In & $1.0 \mathrm{E}+4$ & NCRP96 & NCRP96 & $98 \mathrm{Cf}$ & $2.5 \mathrm{E}+1$ & KS92 & St86 \\
\hline
\end{tabular}


Table 2.11. Concentration Ratios for Saltwater Fish, as Compiled in GENII (Na88) ${ }^{(a)}(\mathrm{Bq} / \mathrm{kg}$ per Bq/L)

\begin{tabular}{|c|c|c|c|}
\hline $\mathrm{Z}$ and Element & $\begin{array}{l}\text { Recommended Value } \\
\text { (Wet Weight) }\end{array}$ & $\mathrm{Z}$ and Element & $\begin{array}{l}\text { Recommended Value } \\
\text { (Wet Weight) }\end{array}$ \\
\hline $02 \mathrm{He}$ & $1.0 \mathrm{E}+0$ & $51 \mathrm{Sb}$ & $1.0 \mathrm{E}+3$ \\
\hline $04 \mathrm{Be}$ & $1.0 \mathrm{E}+3$ & $52 \mathrm{Te}$ & $1.0 \mathrm{E}+3$ \\
\hline $06 \mathrm{C}$ & $2.0 \mathrm{E}+4$ & $53 \mathrm{I}$ & $1.0 \mathrm{E}+1$ \\
\hline $07 \mathrm{~N}$ & $1.0 \mathrm{E}+0$ & $55 \mathrm{Cs}$ & $1.0 \mathrm{E}+2$ \\
\hline $09 \mathrm{~F}$ & $4.0 \mathrm{E}+0$ & $56 \mathrm{Ba}$ & $1.0 \mathrm{E}+1$ \\
\hline $11 \mathrm{Na}$ & $1.0 \mathrm{E}+0$ & $57 \mathrm{La}$ & $3.0 \mathrm{E}+1$ \\
\hline $14 \mathrm{Si}$ & $5.0 \mathrm{E}+4$ & $58 \mathrm{Ce}$ & $1.0 \mathrm{E}+2$ \\
\hline $15 \mathrm{P}$ & $2.8 \mathrm{E}+4$ & $59 \mathrm{Pr}$ & $1.0 \mathrm{E}+2$ \\
\hline $16 \mathrm{~S}$ & $2.0 \mathrm{E}+0$ & $60 \mathrm{Nd}$ & $1.0 \mathrm{E}+2$ \\
\hline $17 \mathrm{Cl}$ & $1.0 \mathrm{E}+0$ & $61 \mathrm{Pm}$ & $5.0 \mathrm{E}+2$ \\
\hline $20 \mathrm{Ca}$ & $2.0 \mathrm{E}+0$ & $62 \mathrm{Sm}$ & $5.0 \mathrm{E}+2$ \\
\hline $21 \mathrm{Sc}$ & $7.5 \mathrm{E}+2$ & $63 \mathrm{Eu}$ & $3.0 \mathrm{E}+2$ \\
\hline $24 \mathrm{Cr}$ & $6.0 \mathrm{E}+2$ & $64 \mathrm{Gd}$ & $5.0 \mathrm{E}+2$ \\
\hline $25 \mathrm{Mn}$ & $4.0 \mathrm{E}+2$ & $65 \mathrm{~Tb}$ & $6.0 \mathrm{E}+1$ \\
\hline $26 \mathrm{Fe}$ & $3.0 \mathrm{E}+3$ & 66 Dy & $5.0 \mathrm{E}+2$ \\
\hline $27 \mathrm{Co}$ & $1.0 \mathrm{E}+2$ & $67 \mathrm{Ho}$ & $3.0 \mathrm{E}+2$ \\
\hline $28 \mathrm{Ni}$ & $1.0 \mathrm{E}+2$ & $68 \mathrm{Er}$ & $1.0 \mathrm{E}+2$ \\
\hline $29 \mathrm{Cu}$ & $1.0 \mathrm{E}+3$ & $72 \mathrm{Hf}$ & $4.0 \mathrm{E}+1$ \\
\hline $30 \mathrm{Zn}$ & $1.0 \mathrm{E}+3$ & $73 \mathrm{Ta}$ & $6.0 \mathrm{E}+1$ \\
\hline $31 \mathrm{Ga}$ & $1.0 \mathrm{e}+3$ & $74 \mathrm{~W}$ & $1.0 \mathrm{E}+1$ \\
\hline $33 \mathrm{As}$ & $3.0 \mathrm{E}+2$ & $77 \mathrm{Ir}$ & $2.0 \mathrm{E}+1$ \\
\hline $34 \mathrm{Se}$ & $6.0 \mathrm{E}+3$ & $80 \mathrm{Hg}$ & $2.0 \mathrm{E}+4$ \\
\hline $35 \mathrm{Br}$ & $1.0 \mathrm{E}+1$ & $81 \mathrm{Tl}$ & $5.0 \mathrm{E}+3$ \\
\hline $37 \mathrm{Rb}$ & $5.0 \mathrm{E}+2$ & $82 \mathrm{~Pb}$ & $2.0 \mathrm{E}+2$ \\
\hline $38 \mathrm{Sr}$ & $4.0 \mathrm{E}+0$ & $83 \mathrm{Bi}$ & $1.5 \mathrm{E}+1$ \\
\hline $39 \mathrm{Y}$ & $2.0 \mathrm{E}+1$ & 84 Po & $2.0 \mathrm{E}+3$ \\
\hline $40 \mathrm{Zr}$ & $5.0 \mathrm{E}+1$ & $88 \mathrm{Ra}$ & $9.5 \mathrm{E}+2$ \\
\hline $41 \mathrm{Nb}$ & $1.0 \mathrm{E}+2$ & $89 \mathrm{Ac}$ & $3.0 \mathrm{E}+1$ \\
\hline $42 \mathrm{Mo}$ & $4.0 \mathrm{E}+1$ & $90 \mathrm{Th}$ & $6.0 \mathrm{E}+2$ \\
\hline $43 \mathrm{Tc}$ & $3.0 \mathrm{E}+1$ & $91 \mathrm{~Pa}$ & $3.0 \mathrm{E}+2$ \\
\hline $44 \mathrm{Ru}$ & $1.0 \mathrm{E}+1$ & $92 \mathrm{U}$ & $5.0 \mathrm{E}+1$ \\
\hline $45 \mathrm{Rh}$ & $1.0 \mathrm{E}+1$ & $93 \mathrm{~Np}$ & $2.5 \mathrm{E}+3$ \\
\hline $46 \mathrm{Pd}$ & $5.0 \mathrm{E}+1$ & $94 \mathrm{Pu}$ & $1.0 \mathrm{E}+3$ \\
\hline $47 \mathrm{Ag}$ & $3.0 \mathrm{E}+2$ & $95 \mathrm{Am}$ & $2.5 \mathrm{E}+3$ \\
\hline $48 \mathrm{Cd}$ & $2.0 \mathrm{E}+3$ & $96 \mathrm{Cm}$ & $2.5 \mathrm{E}+3$ \\
\hline $49 \mathrm{In}$ & $1.0 \mathrm{E}+3$ & $98 \mathrm{Cf}$ & $5.0 \mathrm{E}+1$ \\
\hline $50 \mathrm{Sn}$ & $5.0 \mathrm{E}+4$ & & \\
\hline
\end{tabular}

(a) Concentration ratios for the following elements are not included in the GENII documentation and are not included above: $\mathrm{O}, \mathrm{Mg}, \mathrm{K}, \mathrm{Re}, \mathrm{Os}$, and $\mathrm{Au}$. 
Table 2.12. Concentration Ratios for Saltwater and Freshwater Crustaceans, as Compiled in GENII $(\mathrm{Na} 88)^{(\mathrm{a})}(\mathrm{Bq} / \mathrm{kg}$ muscle per $\mathrm{Bq} / \mathrm{L}$ [wet])

\begin{tabular}{|c|c|c|c|c|c|}
\hline $\begin{array}{c}\mathrm{Z} \text { and } \\
\text { Element }\end{array}$ & Saltwater & Freshwater & $\begin{array}{c}\mathrm{Z} \text { and } \\
\text { Element }\end{array}$ & Saltwater & Freshwater \\
\hline $02 \mathrm{He}$ & $1.0 \mathrm{E}+0$ & $1.0 \mathrm{E}+0$ & $51 \mathrm{Sb}$ & $1.0 \mathrm{E}+2$ & $1.0 \mathrm{E}+2$ \\
\hline $04 \mathrm{Be}$ & $1.0 \mathrm{E}+4$ & $5.0 \mathrm{E}+1$ & $52 \mathrm{Te}$ & $1.0 \mathrm{E}+3$ & $6.1 \mathrm{E}+3$ \\
\hline $06 \mathrm{C}$ & $2.0 \mathrm{E}+4$ & $9.0 \mathrm{E}+3$ & $53 \mathrm{I}$ & $5.0 \mathrm{E}+1$ & $1.0 \mathrm{E}+2$ \\
\hline $07 \mathrm{~N}$ & $1.0 \mathrm{E}+0$ & $1.0 \mathrm{E}+0$ & $55 \mathrm{Cs}$ & $3.0 \mathrm{E}+1$ & $5.0 \mathrm{E}+2$ \\
\hline $09 \mathrm{~F}$ & $4.0 \mathrm{E}+0$ & $1.0 \mathrm{E}+2$ & $56 \mathrm{Ba}$ & $1.0 \mathrm{E}+0$ & $2.0 \mathrm{E}+2$ \\
\hline $11 \mathrm{Na}$ & $1.0 \mathrm{E}-0$ & $1.0 \mathrm{E}+2$ & $57 \mathrm{La}$ & $1.0 \mathrm{E}+2$ & $1.0 \mathrm{E}+3$ \\
\hline $14 \mathrm{Si}$ & $5.0 \mathrm{E}+4$ & $1.0 \mathrm{E}+4$ & $58 \mathrm{Ce}$ & $5.0 \mathrm{E}+2$ & $1.0 \mathrm{E}+3$ \\
\hline $15 \mathrm{P}$ & $3.8 \mathrm{E}+4$ & $1.0 \mathrm{E}+5$ & $59 \operatorname{Pr}$ & $1.0 \mathrm{E}+3$ & $1.0 \mathrm{E}+3$ \\
\hline $16 \mathrm{~S}$ & $1.0 \mathrm{E}+0$ & $1.0 \mathrm{E}+2$ & $61 \mathrm{Pm}$ & $1.0 \mathrm{E}+3$ & $3.0 \mathrm{E}+3$ \\
\hline $17 \mathrm{Cl}$ & $1.0 \mathrm{E}+0$ & $5.0 \mathrm{E}+1$ & $62 \mathrm{Sm}$ & $1.0 \mathrm{E}+3$ & $3.0 \mathrm{E}+3$ \\
\hline $20 \mathrm{Ca}$ & $5.0 \mathrm{E}+0$ & $2.0 \mathrm{E}+3$ & $63 \mathrm{Eu}$ & $1.0 \mathrm{E}+3$ & $3.0 \mathrm{E}+3$ \\
\hline $21 \mathrm{Sc}$ & $3.0 \mathrm{E}+2$ & $1.0 \mathrm{E}+3$ & $64 \mathrm{Gd}$ & $2.0 \mathrm{E}+3$ & $2.0 \mathrm{E}+3$ \\
\hline $24 \mathrm{Cr}$ & $5.0 \mathrm{E}+2$ & $2.0 \mathrm{E}+3$ & $65 \mathrm{~Tb}$ & $1.0 \mathrm{E}+3$ & $1.0 \mathrm{E}+3$ \\
\hline $25 \mathrm{Mn}$ & $8.0 \mathrm{E}+2$ & $1.0 \mathrm{E}+5$ & 66 Dy & $1.0 \mathrm{E}+3$ & $1.0 \mathrm{E}+3$ \\
\hline $26 \mathrm{Fe}$ & $5.0 \mathrm{E}+3$ & $1.0 \mathrm{E}+2$ & $67 \mathrm{Ho}$ & $1.0 \mathrm{E}+3$ & $3.0 \mathrm{E}+3$ \\
\hline $27 \mathrm{Co}$ & $2.0 \mathrm{E}+3$ & $2.0 \mathrm{E}+3$ & $68 \mathrm{Er}$ & $5.0 \mathrm{E}+2$ & $1.0 \mathrm{E}+3$ \\
\hline $28 \mathrm{Ni}$ & $5.0 \mathrm{E}+2$ & $5.0 \mathrm{E}+2$ & $72 \mathrm{Hf}$ & $1.0 \mathrm{E}+3$ & $1.0 \mathrm{E}+3$ \\
\hline $29 \mathrm{Cu}$ & $5.0 \mathrm{E}+3$ & $4.0 \mathrm{E}+2$ & $73 \mathrm{Ta}$ & $3.0 \mathrm{E}+3$ & $3.0 \mathrm{E}+3$ \\
\hline $30 \mathrm{Zn}$ & $5.0 \mathrm{E}+4$ & $1.0 \mathrm{E}+4$ & $74 \mathrm{~W}$ & $1.0 \mathrm{E}+1$ & $1.0 \mathrm{E}+1$ \\
\hline $31 \mathrm{Ga}$ & $1.0 \mathrm{E}+4$ & $1.0 \mathrm{E}+4$ & $77 \mathrm{Ir}$ & $1.0 \mathrm{E}+2$ & $2.0 \mathrm{E}+2$ \\
\hline $33 \mathrm{As}$ & $3.0 \mathrm{E}+2$ & $3.0 \mathrm{E}+2$ & $80 \mathrm{Hg}$ & $2.0 \mathrm{E}+4$ & $2.0 \mathrm{E}+4$ \\
\hline $34 \mathrm{Se}$ & $5.0 \mathrm{E}+3$ & $2.0 \mathrm{E}+3$ & $81 \mathrm{Tl}$ & $1.0 \mathrm{E}+3$ & $1.0 \mathrm{E}+3$ \\
\hline $35 \mathrm{Br}$ & $1.0 \mathrm{E}+1$ & $3.3 \mathrm{E}+2$ & $82 \mathrm{~Pb}$ & $1.0 \mathrm{E}+3$ & $5.0 \mathrm{E}+2$ \\
\hline $37 \mathrm{Rb}$ & $2.0 \mathrm{E}+2$ & $1.0 \mathrm{E}+3$ & $83 \mathrm{Bi}$ & $1.0 \mathrm{E}+3$ & $1.0 \mathrm{E}+5$ \\
\hline $38 \mathrm{Sr}$ & $1.0 \mathrm{E}+0$ & $1.0 \mathrm{E}+2$ & $84 \mathrm{Po}$ & $5.0 \mathrm{E}+4$ & $2.0 \mathrm{E}+4$ \\
\hline $39 \mathrm{Y}$ & $1.0 \mathrm{E}+3$ & $1.0 \mathrm{E}+3$ & $88 \mathrm{Ra}$ & $1.0 \mathrm{E}+2$ & $1.0 \mathrm{E}+3$ \\
\hline $40 \mathrm{Zr}$ & $5.0 \mathrm{E}+1$ & $5.0 \mathrm{E}+1$ & $89 \mathrm{Ac}$ & $1.0 \mathrm{E}+3$ & $1.0 \mathrm{E}+3$ \\
\hline $41 \mathrm{Nb}$ & $5.0 \mathrm{E}+1$ & $5.0 \mathrm{E}+1$ & $90 \mathrm{Th}$ & $1.0 \mathrm{E}+3$ & $1.0 \mathrm{E}+2$ \\
\hline $42 \mathrm{Mo}$ & $2.0 \mathrm{E}+1$ & $1.0 \mathrm{E}+2$ & $91 \mathrm{~Pa}$ & $1.0 \mathrm{E}+1$ & $3.0 \mathrm{E}+1$ \\
\hline $43 \mathrm{Tc}$ & $1.0 \mathrm{E}+1$ & $1.0 \mathrm{E}+2$ & $92 \mathrm{U}$ & $1.0 \mathrm{E}+1$ & $1.0 \mathrm{E}+2$ \\
\hline $44 \mathrm{Ru}$ & $1.0 \mathrm{E}+2$ & $3.0 \mathrm{E}+2$ & $93 \mathrm{~Np}$ & $1.0 \mathrm{E}+1$ & $3.0 \mathrm{E}+1$ \\
\hline $45 \mathrm{Rh}$ & $1.0 \mathrm{E}+2$ & $3.0 \mathrm{E}+2$ & $94 \mathrm{Pu}$ & $3.0 \mathrm{E}+2$ & $1.0 \mathrm{E}+2$ \\
\hline $46 \mathrm{Pd}$ & $3.0 \mathrm{E}+2$ & $2.0 \mathrm{E}+3$ & $95 \mathrm{Am}$ & $3.6 \mathrm{E}+2$ & $1.0 \mathrm{E}+2$ \\
\hline $47 \mathrm{Ag}$ & $3.5 \mathrm{E}+2$ & $2.0 \mathrm{E}+2$ & $96 \mathrm{Cm}$ & $4.6 \mathrm{E}+2$ & $1.0 \mathrm{E}+3$ \\
\hline $48 \mathrm{Cd}$ & $5.0 \mathrm{E}+3$ & $1.0 \mathrm{E}+4$ & $98 \mathrm{Cf}$ & $5.0 \mathrm{E}+2$ & $1.0 \mathrm{E}+3$ \\
\hline 49 In & $1.0 \mathrm{E}+4$ & $1.0 \mathrm{E}+4$ & & & \\
\hline $50 \mathrm{Sn}$ & $5.0 \mathrm{E}+4$ & $1.0 \mathrm{E}+4$ & & & \\
\hline
\end{tabular}

(a) Concentration ratios for the following elements are not included in the GENII documentation and are not included above: $\mathrm{O}, \mathrm{Mg}, \mathrm{K}, \mathrm{Nd}, \mathrm{Re}, \mathrm{Os}$, and $\mathrm{Au}$. 
Table 2.13. Concentration Ratios for Molluscs, as Compiled in GENII $(\mathrm{Na} 88)^{(\mathrm{a})}(\mathrm{Bq} / \mathrm{kg}$ per Bq/L)

\begin{tabular}{|c|c|c|c|c|c|}
\hline $\begin{array}{c}\mathrm{Z} \text { and } \\
\text { Element }\end{array}$ & Saltwater & Freshwater & $\begin{array}{c}\mathrm{Z} \text { and } \\
\text { Element }\end{array}$ & Saltwater & Freshwater \\
\hline$\overline{02 \mathrm{He}}$ & $1.0 \mathrm{E}+0$ & $1.0 \mathrm{E}+0$ & $51 \mathrm{Sb}$ & $1.2 \mathrm{E}+3$ & $1.0 \mathrm{E}+2$ \\
\hline $04 \mathrm{Be}$ & $1.0 \mathrm{E}+4$ & $5.0 \mathrm{E}+1$ & $52 \mathrm{Te}$ & $1.0 \mathrm{E}+3$ & $6.1 \mathrm{E}+3$ \\
\hline $06 \mathrm{C}$ & $2.0 \mathrm{E}+4$ & $9.0 \mathrm{E}+3$ & $53 \mathrm{I}$ & $5.0 \mathrm{E}+1$ & $1.0 \mathrm{E}+2$ \\
\hline $07 \mathrm{~N}$ & $1.0 \mathrm{E}+0$ & $1.0 \mathrm{E}+0$ & $55 \mathrm{Cs}$ & $3.0 \mathrm{E}+1$ & $5.0 \mathrm{E}+2$ \\
\hline $09 \mathrm{~F}$ & $4.0 \mathrm{E}+0$ & $1.0 \mathrm{E}+2$ & $56 \mathrm{Ba}$ & $2.0 \mathrm{E}+1$ & $2.0 \mathrm{E}+2$ \\
\hline $11 \mathrm{Na}$ & $3.0 \mathrm{E}-0$ & $1.0 \mathrm{E}+2$ & $57 \mathrm{La}$ & $1.0 \mathrm{E}+2$ & $1.0 \mathrm{E}+3$ \\
\hline $14 \mathrm{Si}$ & $5.0 \mathrm{E}+4$ & $1.0 \mathrm{E}+4$ & $58 \mathrm{Ce}$ & $5.0 \mathrm{E}+2$ & $1.0 \mathrm{E}+3$ \\
\hline $15 \mathrm{P}$ & $4.5 \mathrm{E}+4$ & $1.0 \mathrm{E}+5$ & $59 \operatorname{Pr}$ & $1.0 \mathrm{E}+3$ & $1.0 \mathrm{E}+3$ \\
\hline $16 \mathrm{~S}$ & $4.0 \mathrm{E}+0$ & $1.0 \mathrm{E}+2$ & $61 \mathrm{Pm}$ & $5.0 \mathrm{E}+3$ & $3.0 \mathrm{E}+3$ \\
\hline $17 \mathrm{Cl}$ & $1.0 \mathrm{E}+0$ & $5.0 \mathrm{E}+1$ & $62 \mathrm{Sm}$ & $5.0 \mathrm{E}+3$ & $3.0 \mathrm{E}+3$ \\
\hline $20 \mathrm{Ca}$ & $1.0 \mathrm{E}+0$ & $2.0 \mathrm{E}+3$ & $63 \mathrm{Eu}$ & $7.0 \mathrm{E}+3$ & $3.0 \mathrm{E}+3$ \\
\hline $21 \mathrm{Sc}$ & $1.0 \mathrm{E}+2$ & $1.0 \mathrm{E}+3$ & $64 \mathrm{Gd}$ & $5.0 \mathrm{E}+3$ & $5.0 \mathrm{E}+3$ \\
\hline $24 \mathrm{Cr}$ & $1.1 \mathrm{E}+3$ & $2.0 \mathrm{E}+3$ & $65 \mathrm{~Tb}$ & $3.0 \mathrm{E}+3$ & $1.0 \mathrm{E}+3$ \\
\hline $25 \mathrm{Mn}$ & $6.0 \mathrm{E}+3$ & $1.0 \mathrm{E}+5$ & 66 Dy & $5.0 \mathrm{E}+3$ & $5.0 \mathrm{E}+3$ \\
\hline $26 \mathrm{Fe}$ & $3.0 \mathrm{E}+4$ & $1.0 \mathrm{E}+2$ & $67 \mathrm{Ho}$ & $1.0 \mathrm{E}+3$ & $3.0 \mathrm{E}+3$ \\
\hline $27 \mathrm{Co}$ & $3.0 \mathrm{E}+3$ & $2.0 \mathrm{E}+3$ & $68 \mathrm{Er}$ & $5.0 \mathrm{E}+2$ & $1.0 \mathrm{E}+3$ \\
\hline $28 \mathrm{Ni}$ & $5.0 \mathrm{E}+2$ & $5.0 \mathrm{E}+2$ & $72 \mathrm{Hf}$ & $3.0 \mathrm{E}+3$ & $3.0 \mathrm{E}+3$ \\
\hline $29 \mathrm{Cu}$ & $5.0 \mathrm{E}+3$ & $4.0 \mathrm{E}+2$ & $73 \mathrm{Ta}$ & $3.0 \mathrm{E}+3$ & $3.0 \mathrm{E}+3$ \\
\hline $30 \mathrm{Zn}$ & $3.0 \mathrm{E}+4$ & $1.0 \mathrm{E}+4$ & $74 \mathrm{~W}$ & $1.0 \mathrm{E}+2$ & $1.0 \mathrm{E}+1$ \\
\hline $31 \mathrm{Ga}$ & $1.0 \mathrm{E}+4$ & $1.0 \mathrm{E}+4$ & $77 \mathrm{Ir}$ & $1.0 \mathrm{E}+2$ & $2.0 \mathrm{E}+2$ \\
\hline $33 \mathrm{As}$ & $3.0 \mathrm{E}+2$ & $3.0 \mathrm{E}+2$ & $80 \mathrm{Hg}$ & $1.0 \mathrm{E}+4$ & $2.0 \mathrm{E}+4$ \\
\hline $34 \mathrm{Se}$ & $6.0 \mathrm{E}+3$ & $2.0 \mathrm{E}+3$ & $81 \mathrm{Tl}$ & $5.0 \mathrm{E}+3$ & $5.0 \mathrm{E}+3$ \\
\hline $35 \mathrm{Br}$ & $1.0 \mathrm{E}+1$ & $3.3 \mathrm{E}+2$ & $82 \mathrm{~Pb}$ & $1.0 \mathrm{E}+3$ & $5.0 \mathrm{E}+2$ \\
\hline $37 \mathrm{Rb}$ & $2.0 \mathrm{E}+2$ & $1.0 \mathrm{E}+3$ & $83 \mathrm{Bi}$ & $1.0 \mathrm{E}+3$ & $1.0 \mathrm{E}+5$ \\
\hline $38 \mathrm{Sr}$ & $4.0 \mathrm{E}+1$ & $1.0 \mathrm{E}+2$ & $84 \mathrm{Po}$ & $1.0 \mathrm{E}+4$ & $2.0 \mathrm{E}+4$ \\
\hline $39 \mathrm{Y}$ & $1.0 \mathrm{E}+3$ & $1.0 \mathrm{E}+3$ & $88 \mathrm{Ra}$ & $1.0 \mathrm{E}+2$ & $1.0 \mathrm{E}+3$ \\
\hline $40 \mathrm{Zr}$ & $5.0 \mathrm{E}+1$ & $5.0 \mathrm{E}+1$ & 89 Ac & $1.0 \mathrm{E}+3$ & $1.0 \mathrm{E}+3$ \\
\hline $41 \mathrm{Nb}$ & $5.0 \mathrm{E}+1$ & $5.0 \mathrm{E}+1$ & $90 \mathrm{Th}$ & $1.0 \mathrm{E}+3$ & $1.0 \mathrm{E}+2$ \\
\hline 42 Mo & $2.0 \mathrm{E}+1$ & $1.0 \mathrm{E}+2$ & $91 \mathrm{~Pa}$ & $1.0 \mathrm{E}+1$ & $3.0 \mathrm{E}+1$ \\
\hline $43 \mathrm{Tc}$ & $2.0 \mathrm{E}+1$ & $1.0 \mathrm{E}+2$ & $92 \mathrm{U}$ & $3.0 \mathrm{E}+1$ & $1.0 \mathrm{E}+2$ \\
\hline $44 \mathrm{Ru}$ & $2.0 \mathrm{E}+3$ & $3.0 \mathrm{E}+2$ & $93 \mathrm{~Np}$ & $1.5 \mathrm{E}+2$ & $3.0 \mathrm{E}+1$ \\
\hline $45 \mathrm{Rh}$ & $1.0 \mathrm{E}+2$ & $3.0 \mathrm{E}+2$ & $94 \mathrm{Pu}$ & $3.0 \mathrm{E}+3$ & $1.0 \mathrm{E}+2$ \\
\hline $46 \mathrm{Pd}$ & $3.0 \mathrm{E}+2$ & $2.0 \mathrm{E}+3$ & $95 \mathrm{Am}$ & $2.9 \mathrm{E}+2$ & $1.0 \mathrm{E}+2$ \\
\hline $47 \mathrm{Ag}$ & $5.8 \mathrm{E}+2$ & $2.0 \mathrm{E}+2$ & $96 \mathrm{Cm}$ & $4.6 \mathrm{E}+2$ & $1.0 \mathrm{E}+3$ \\
\hline $48 \mathrm{Cd}$ & $1.0 \mathrm{E}+4$ & $1.0 \mathrm{E}+4$ & $98 \mathrm{Cf}$ & $2.0 \mathrm{E}+3$ & $1.0 \mathrm{E}+3$ \\
\hline 49 In & $1.0 \mathrm{E}+4$ & $1.0 \mathrm{E}+4$ & & & \\
\hline $50 \mathrm{Sn}$ & $5.0 \mathrm{E}+4$ & $1.0 \mathrm{E}+4$ & & & \\
\hline
\end{tabular}

(a) Concentration ratios for the following elements are not included in the GENII documentation and are not included above: $\mathrm{O}, \mathrm{Mg}, \mathrm{K}, \mathrm{Nd}, \mathrm{Re}, \mathrm{Os}$, and $\mathrm{Au}$. 
Table 2.14. Concentration Ratios for Water Plants, as Compiled in GENII $(\mathrm{Na} 88)^{(\mathrm{a})}(\mathrm{Bq} / \mathrm{kg}$ per Bq/L)

\begin{tabular}{|c|c|c|c|c|c|}
\hline $\begin{array}{c}\mathrm{Z} \text { and } \\
\text { Element }\end{array}$ & Saltwater & Freshwater & $\begin{array}{c}\mathrm{Z} \text { and } \\
\text { Element }\end{array}$ & Saltwater & Freshwater \\
\hline $02 \mathrm{He}$ & $1.0 \mathrm{E}+0$ & $1.0 \mathrm{E}+0$ & $51 \mathrm{Sb}$ & $1.0 \mathrm{E}+2$ & $1.0 \mathrm{E}+3$ \\
\hline $04 \mathrm{Be}$ & $1.0 \mathrm{E}+4$ & $2.0 \mathrm{E}+2$ & $52 \mathrm{Te}$ & $4.0 \mathrm{E}+2$ & $1.0 \mathrm{E}+2$ \\
\hline $06 \mathrm{C}$ & $1.8 \mathrm{E}+3$ & $4.5 \mathrm{E}+3$ & $53 \mathrm{I}$ & $1.5 \mathrm{E}+3$ & $3.0 \mathrm{E}+2$ \\
\hline $07 \mathrm{~N}$ & $1.0 \mathrm{E}+0$ & $1.0 \mathrm{E}+0$ & $55 \mathrm{Cs}$ & $7.0 \mathrm{E}+2$ & $1.0 \mathrm{E}+3$ \\
\hline $09 \mathrm{~F}$ & $1.0 \mathrm{E}+0$ & $2.0 \mathrm{E}+0$ & $56 \mathrm{Ba}$ & $1.0 \mathrm{E}+2$ & $5.0 \mathrm{E}+2$ \\
\hline $11 \mathrm{Na}$ & $1.0 \mathrm{E}+0$ & $1.0 \mathrm{E}+2$ & $57 \mathrm{La}$ & $3.0 \mathrm{E}+2$ & $5.0 \mathrm{E}+3$ \\
\hline $14 \mathrm{Si}$ & $5.0 \mathrm{E}+4$ & $5.0 \mathrm{E}+4$ & $58 \mathrm{Ce}$ & $5.0 \mathrm{E}+3$ & $4.0 \mathrm{E}+3$ \\
\hline $15 \mathrm{P}$ & $1.0 \mathrm{E}+5$ & $5.0 \mathrm{E}+5$ & $59 \mathrm{Pr}$ & $1.0 \mathrm{E}+3$ & $5.0 \mathrm{E}+3$ \\
\hline $16 \mathrm{~S}$ & $5.0 \mathrm{E}+0$ & $1.0 \mathrm{E}+0$ & $61 \mathrm{Pm}$ & $3.0 \mathrm{E}+3$ & $5.0 \mathrm{E}+3$ \\
\hline $17 \mathrm{Cl}$ & $1.0 \mathrm{E}+0$ & $5.0 \mathrm{E}+1$ & $62 \mathrm{Sm}$ & $5.0 \mathrm{E}+3$ & $5.0 \mathrm{E}+3$ \\
\hline $20 \mathrm{Ca}$ & $5.0 \mathrm{E}+1$ & $1.0 \mathrm{E}+3$ & $63 \mathrm{Eu}$ & $5.0 \mathrm{E}+3$ & $5.0 \mathrm{E}+3$ \\
\hline $21 \mathrm{Sc}$ & $1.0 \mathrm{E}+3$ & $1.0 \mathrm{E}+4$ & $64 \mathrm{Gd}$ & $1.0 \mathrm{E}+0$ & $1.0 \mathrm{E}+0$ \\
\hline $24 \mathrm{Cr}$ & $4.0 \mathrm{E}+4$ & $4.0 \mathrm{E}+4$ & $65 \mathrm{~Tb}$ & $1.0 \mathrm{E}+3$ & $5.0 \mathrm{E}+3$ \\
\hline $25 \mathrm{Mn}$ & $1.0 \mathrm{E}+4$ & $1.0 \mathrm{E}+4$ & 66 Dy & $1.0 \mathrm{E}+0$ & $1.0 \mathrm{E}+0$ \\
\hline $26 \mathrm{Fe}$ & $5.0 \mathrm{E}+4$ & $1.0 \mathrm{E}+3$ & $67 \mathrm{Ho}$ & $5.0 \mathrm{E}+3$ & $5.0 \mathrm{E}+3$ \\
\hline $27 \mathrm{Co}$ & $4.0 \mathrm{E}+2$ & $1.0 \mathrm{E}+3$ & $68 \mathrm{Er}$ & $5.0 \mathrm{E}+3$ & $4.0 \mathrm{E}+3$ \\
\hline $28 \mathrm{Ni}$ & $3.0 \mathrm{E}+3$ & $5.0 \mathrm{E}+2$ & $72 \mathrm{Hf}$ & $1.0 \mathrm{E}+0$ & $1.0 \mathrm{E}+0$ \\
\hline $29 \mathrm{Cu}$ & $1.0 \mathrm{E}+3$ & $2.0 \mathrm{E}+3$ & $73 \mathrm{Ta}$ & $1.0 \mathrm{E}+0$ & $1.0 \mathrm{E}+0$ \\
\hline $30 \mathrm{Zn}$ & $5.0 \mathrm{E}+4$ & $2.0 \mathrm{E}+4$ & $74 \mathrm{~W}$ & $1.0 \mathrm{E}+2$ & $1.2 \mathrm{E}+3$ \\
\hline $31 \mathrm{Ga}$ & $1.0 \mathrm{E}+0$ & $1.0 \mathrm{E}+0$ & $77 \mathrm{Ir}$ & $1.0 \mathrm{E}+2$ & $2.0 \mathrm{E}+2$ \\
\hline $33 \mathrm{As}$ & $3.0 \mathrm{E}+2$ & $3.0 \mathrm{E}+2$ & $80 \mathrm{Hg}$ & $3.4 \mathrm{E}+4$ & $3.4 \mathrm{E}+4$ \\
\hline $34 \mathrm{Se}$ & $1.0 \mathrm{E}+3$ & $1.0 \mathrm{E}+2$ & $81 \mathrm{Tl}$ & $1.0 \mathrm{E}+0$ & $1.0 \mathrm{E}+0$ \\
\hline $35 \mathrm{Br}$ & $1.0 \mathrm{E}+1$ & $5.0 \mathrm{E}+1$ & $82 \mathrm{~Pb}$ & $5.0 \mathrm{E}+4$ & $2.0 \mathrm{E}+3$ \\
\hline $37 \mathrm{Rb}$ & $1.0 \mathrm{E}+3$ & $1.0 \mathrm{E}+3$ & $83 \mathrm{Bi}$ & $1.0 \mathrm{E}+4$ & $1.5 \mathrm{E}+3$ \\
\hline $38 \mathrm{Sr}$ & $3.0 \mathrm{E}+2$ & $3.0 \mathrm{E}+3$ & $84 \mathrm{Po}$ & $1.0 \mathrm{E}+3$ & $2.0 \mathrm{E}+3$ \\
\hline $39 \mathrm{Y}$ & $3.0 \mathrm{E}+2$ & $5.0 \mathrm{E}+3$ & $88 \mathrm{Ra}$ & $1.0 \mathrm{E}+3$ & $3.0 \mathrm{E}+4$ \\
\hline $40 \mathrm{Zr}$ & $5.0 \mathrm{E}+3$ & $5.0 \mathrm{E}+3$ & 89 Ac & $1.0 \mathrm{E}+3$ & $1.0 \mathrm{E}+4$ \\
\hline $41 \mathrm{Nb}$ & $5.0 \mathrm{E}+2$ & $5.0 \mathrm{E}+2$ & $90 \mathrm{Th}$ & $2.0 \mathrm{E}+3$ & $3.0 \mathrm{E}+3$ \\
\hline 42 Mo & $1.0 \mathrm{E}+2$ & $1.0 \mathrm{E}+3$ & $91 \mathrm{~Pa}$ & $5.0 \mathrm{E}+1$ & $3.0 \mathrm{E}+2$ \\
\hline $43 \mathrm{Tc}$ & $5.0 \mathrm{E}+3$ & $5.0 \mathrm{E}+3$ & $92 \mathrm{U}$ & $1.0 \mathrm{E}+3$ & $9.0 \mathrm{E}+2$ \\
\hline $44 \mathrm{Ru}$ & $1.0 \mathrm{E}+3$ & $2.0 \mathrm{E}+3$ & $93 \mathrm{~Np}$ & $6.0 \mathrm{E}+0$ & $3.0 \mathrm{E}+2$ \\
\hline $45 \mathrm{Rh}$ & $1.0 \mathrm{E}+2$ & $2.0 \mathrm{E}+2$ & $94 \mathrm{Pu}$ & $3.6 \mathrm{E}+3$ & $8.9 \mathrm{E}+2$ \\
\hline $46 \mathrm{Pd}$ & $2.0 \mathrm{E}+4$ & $2.0 \mathrm{E}+3$ & $95 \mathrm{Am}$ & $2.9 \mathrm{E}+3$ & $3.0 \mathrm{E}+3$ \\
\hline $47 \mathrm{Ag}$ & $1.0 \mathrm{E}+3$ & $1.0 \mathrm{E}+3$ & $96 \mathrm{Cm}$ & $4.3 \mathrm{E}+4$ & $1.0 \mathrm{E}+4$ \\
\hline $48 \mathrm{Cd}$ & $1.0 \mathrm{E}+3$ & $5.0 \mathrm{E}+2$ & $98 \mathrm{Cf}$ & $5.0 \mathrm{E}+3$ & $5.0 \mathrm{E}+3$ \\
\hline 49 In & $1.0 \mathrm{E}+0$ & $1.0 \mathrm{E}+0$ & & & \\
\hline $50 \mathrm{Sn}$ & $5.0 \mathrm{E}+4$ & $5.0 \mathrm{E}+4$ & & & \\
\hline
\end{tabular}

(a) Concentration ratios for the following elements are not included in the GENII documentation and are not included above: $\mathrm{O}, \mathrm{Mg}, \mathrm{K}, \mathrm{Nd}, \mathrm{Re}, \mathrm{Os}$, and $\mathrm{Au}$. 


\subsection{Poultry}

The N, F, S, K, Re, Os, Au, Bi, and Po transfer factors for feed-to-poultry are derived from the geometric mean of the other available transfer factors in their respective groups. The $\mathrm{Cr}, \mathrm{Rh}, \mathrm{Ta}, \mathrm{W}$, and Ir poultry transfer factors are set equal to a single established value in their respective chemical groups. The transfer factors for $\mathrm{Ga}, \mathrm{In}, \mathrm{Tl}, \mathrm{Si}, \mathrm{Sn}$, and $\mathrm{Pb}$ are based on the greatest value (most conservative) of the next periodic table group (VA) of elements. For poultry, this resulted in a value of 0.8 being assigned to the missing values. All the lanthanide elements are assigned the value for Ce. Those actinides without a value are assigned the value for Am.

\subsection{Milk}

The Ta, Hf, and Cf transfer factors for feed-to-milk are set equal to the values from $\mathrm{Nb}, \mathrm{Zr}$, and $\mathrm{Am}$, respectively. All the lanthanides are based on Ce values. All other values are based on the recommended values in a compilation document.

\subsection{Egg}

The N, F, S, Cl, Sc, As, Re, Os, Hg, Bi, Po, and Ra transfer factors for feed-to-eggs are calculated as the geometric mean of the established transfer factors in their respective chemical groups. The $\mathrm{Cr}, \mathrm{Rh}$, $\mathrm{Ag}, \mathrm{Hf}, \mathrm{Ta}, \mathrm{W}, \mathrm{Ir}$, and Au transfer factors for eggs are based on a single established value in their respective groups. The transfer factors for $\mathrm{Ga}, \mathrm{In}, \mathrm{Tl}, \mathrm{Si}, \mathrm{Sn}$, and $\mathrm{Pb}$ are based on the greatest value (most conservative) for the next group (VA) of elements. For eggs, this results in a value of 1.0 being assigned to the missing values. All the lanthanide elements are assigned the value for Ce. Those actinides without a value are assigned the value for Am.

\subsection{Freshwater Fish}

Most of the water-to-fish transfer factors are available from one of the compilations. However, values for all lanthanides are based on Ce.

\subsection{Saltwater Fish, Crustaceans, Molluscs, and Water Plants}

Four additional types of transfer factors are all derived from GENII 1.485 (Na88) without current updating. These are the transfer factors for saltwater fish, fresh and saltwater crustaceans, fresh and saltwater molluscs, and fresh and saltwater plants. All transfer factors in these tables come from the GENII 1.485 data files. For marine species, the GENII 1.485 values in Napier et al. (1988) are largely traceable back to A Model for the Approximate Calculation of Safe Rates of Discharge of Radioactive Wastes into Marine Environments (Fr67). Specifically for freshwater environments, the parameters are ultimately traceable to Concentration Factors of Chemical Elements in Edible Aquatic Organisms (Th72), which in turn is primarily derived from Ng's (Ng68) Handbook for Estimating the Maximum Internal Dose from Radionuclides Released to the Biosphere. 


\subsection{References}

(Ba84) Baes, C.F., R.D. Sharp, A.L. Sjoreen, and R.W. Shor. 1984. A Review and Analysis of Parameters for Assessing Transport of Environmentally Released Radionuclides through Agriculture. ORNL-5786, Oak Ridge National Laboratory, Oak Ridge, Tennessee.

(Bi89) Bishop, G.P., C.J. Beetham, and Y.S. Cuff. 1989. Review of Literature for Chlorine Technetium, Iodine and Neptunium. Nirex Radioactive Waste Disposal Safety Studies, NSS/R193, UK Nirex Ltd., Harwell, England.

(Bi91) Bilo, M. 1991. Untersuchugen zum Transfer des durch den Reaktorunfall von Tschernobyl abgelagerten Radiocasiums vom Boden in die Pflanze. Thesis, Personal Communication w/ Zezina, N. Tubingen.

(Br79) Bruland, W., et al. 1979. "Transfer of organically bound radionuclides through food chains to man. Model--example with radiocobalt and vitamin B12." In Biological Implications of Radionuclides Released from Nuclear Industries. Vol. 2, Proceedings of a Symposium. IAEA, Vienna.

(CEC87) Commission of the European Communities. 1987. Radionuclide Transfer Factors of Animal Feedingstuffs and Animal Products. CEC Doc. 7682/87, Luxembourg.

(CT83) Coughtrey, P.C., and M.C. Thorne. 1983. Radionuclide Distribution and Transport in Terrestrial and Aquatic Ecosystems, A Critical Review of Data. Vols. 1-6. A.A. Balkema, Rotterdam.

(Co90) Coughtrey, P.J. 1990. Radioactivity Transfer to Animal Products. EUR 12608 EN, Commission of the European Communities, Luxembourg.

(Cr90) Cramp, T.J., Y.S. Cuff, A. Davis, and J.E. Morgan. 1990. Review of Data for Uranium, Nickel, and Cobalt. 2150-RI, Associated Nuclear Services, Ltd., Epsom, England.

(En88a) Ennis, M.E., Jr., G.M. Ward, J.E. Johnson, and K.N. Boamah. 1988. "Transfer coefficients of selected radionuclides to animal products II. Hen eggs and meat.” Health Physics, 54:167-170.

(En88b) Ennis, M.E. Jr., J.E. Johnson, G.M. Ward, and G.M. Voigt. 1988. "A specific activity effect in the metabolism of Tc." Health Physics, 54:157-160.

(Fr67) Freke, A.M. 1967. "A model for the approximate calculation of safe rates of discharge of radioactive wastes into marine environments." Health Physics, 13: 743-758.

(Fr82) Frissel, M.J. 1992. An Update of the Recommended Soil-to-Plant Transfer Factors. Eighth Report of the IUR Working Group on Soil-to-Plant Transfer Factors, IUR, Balen, Belgium. 
(Fr89) Frissel, M.J., and K.E. van Bergeijk. 1989. Mean Transfer Values Derived by Simple Statistical Regression Analysis. Sixth Report of IUR Working Group on Soil-to-Plant Transfer Factors, RIVM, Bilthoven, Netherlands.

(Fu78) Furr, A.K., T.F. Parkinson, C.L. Heffron, J.T. Reid, W.M. Haschek, W.H. Gutenmann, C.A. Backe, L.E. St. John, Jr., and D.J. Lisk. 1979. "Elemental content of tissues and excreta of lambs, goats and kids fed white sweet clover growing on fly ash." Journal of Agric. Food Chem., 26(4):847-851.

(Ho91) Holzer, F., and K. Wichterey. 1991. Ermittlung von Transferfaktoren fur den Ubergang naturlicher Radionuklide vom Boden in die Pflanzen im Uranerzbergbaugebiet. Jahresbericht des Bundesamtes fur Strahlenschutz, 146.

(IAEA82) International Atomic Energy Agency. 1982. Generic Models and Parameters for Assessing the Environmental Transfer of Radionuclides from Routine Releases, Exposures of Critical Groups. Safety Series No. 57, IAEA, Vienna, Austria.

(IAEA87) International Atomic Energy Agency. 1987. Draft Working Document: Handbook of Parameter Values for the Prediction of Radionuclide Transfer in the Terrestrial and Freshwater Environments. IAEA, Vienna, Austria.

(IAEA94) International Atomic Energy Agency, International Union of Radioecologists. 1994. Handbook of Parameter Values for the Prediction of Radionuclide Transfer in Temperate Environments. Tech. Rep. Series No. 364, IAEA, Vienna, Austria.

(IUR89) International Union of Radioecologists. 1989. Sixth Report of the Working Group on Soil-toPlant Transfer Factors. RIVM, Bilthoven, The Netherlands.

(Jo88) Johnson, J.E., G.M. Ward, M.E. Ennis, Jr., and K.N. Boamah. 1988. "Transfer coefficients of selected radionuclides to animal products, 1. Comparison of milk and meat from dairy cows and goats." Health Physics, 54:161-166.

(KS92) Kennedy, W.E., Jr., and D.L. Strenge. 1992. Residual Radioactive Contamination from Decommissioning: Technical Basis for Translating Contamination Levels to Annual Total Effective Dose Equivalent. NUREG/CR-5512 (PNL-7994), Pacific Northwest Laboratory, Richland, Washington.

(LS85) Lawson, G., and G.M. Smith. 1985. BIOS: A Model to Predict Radionuclide Transfer and Doses to Man Following Releases for Geological Repositories for Radioactive Wastes. NRPB-R169, National Radiation Protection Board, Chilton, England.

(MB90) Morgan, J.E., and C.J. Beetham. 1990. Review of Literature for Radium, Protactinium, Tin and Carbon. Nirex Radioactive Waste Disposal Safety Studies, UK Nirex LT Harwell, England. 
(Na88) Napier, B.A., R.A. Peloquin, D.L. Strenge, and J.V. Ramsdell. 1988. GENII - The Hanford Environmental Radiation Dosimetry Software System. PNL-6584, Pacific Northwest Laboratory, Richland, Washington.

(NCRP86) National Council on Radiation Protection and Measurements. 1986. Screening Techniques for Determining Compliance with Environmental Standards, Releases of Radionuclides to the Atmosphere. NCRP Commentary No. 3, NCRP, Bethesda, Maryland.

(NCRP96) National Council on Ionizing Radiation and Protection. 1996. Screening Models for Releases of Radionuclides to Atmosphere, Surface Water and Ground. NCRP Report No. 123, Vol. I., NCRP, Bethesda, Maryland.

(Ng68) Ng, Y.C., C.A. Burton, S.E. Thompson, R.K. Tandy, H.K. Kretner, and M.W. Pratt. 1968. "Prediction of the maximum dosage to man from the fallout of nuclear devices." In Handbook for Estimating the Maximum Internal Dose from Radionuclides Released to the Biosphere. UCRL-50163, Pt. IV, Lawrence Radiation Laboratory, Livermore, California.

(Ng77) Ng, Y.C., C.S. Colsher, D.J. Quinn, and S.E. Thompson. 1977. Transfer Coefficients for the Prediction of the Dose to Man via the Forage-Cow-Milk Pathway from Radionuclides Released to the Biosphere. UCRL-51939, Lawrence Livermore National Laboratory, Livermore, California.

(Ng79) Ng, Y.C., C.S. Colsher, and S.E. Thompson. 1979. "Transfer coefficients for assessing the terrestrial food chains - their derivation and limitations." In Radioactivitat und Umwelt, H.J. Kellermann, ed., Vol. I, pp. 455-481. Proceedings of the $12^{\text {th }}$ Annual Conference of the Fachverband fur Strahlenschutz, Nederney, West Germany, 2-6 October, 1978.

(Ng82a) Ng, Y.C., C.S. Colsher, and S.E. Thompson. 1982a. Transfer Coefficients for Assessing the Dose from Radionuclides in Meat and Eggs. NUREG/CR-2976, UCID-19464, Lawrence Livermore National Laboratory, California.

(Ng82b) Ng, Y.C., C.S. Colsher, and S.E. Thompson. 1982b. Soil-to-Plant Concentration Factors for Radiological Assessments. NUREG/CR-2975, UCID19463, Lawrence Livermore National Laboratory, Livermore, California.

(Ng82c) Ng, Y.C. 1982c. "A review of transfer factors for assessing the dose from radionuclides in agricultural products." Nuclear Safety, 23:57-71.

(Sn94) Snyder, S.F., W.T. Farris, B.A. Napier, T.A. Ikenberry, and R.O. Gilbert. 1994. Parameters Used in the Environmental Pathways and Radiological Dose Modules (DESCARTES, CIDER and CRD Codes) of the Hanford Environmental Dose Reconstruction Integrated Codes (HEDRIC). PNWD-2033 HEDR, Rev. 1, Pacific Northwest Laboratories, Richland, Washington. 
(St86) Strenge, D.L., R.A. Peloquin, and G. Whelan. 1986. LADTAP II - Technical Reference and User Guide. NUREG/CR-4013 (PNL-5270), U.S. Nuclear Regulatory Commission, Washington, D.C.

(Th94) Thiede, M.E., D.J. Bates, E.I. Mart, and R.W. Hanf. 1994. A Guide to Environmental Monitoring Data, 1945-1972. PNWD-2226 HEDR, Battelle, Pacific Northwest Laboratories, Richland, Washington.

(Th72) Thompson, S.E., C.A. Burton, D.J. Quinn, and Y.C. Ng. 1972. Concentration Factors of Chemical Elements in Edible Aquatic Organisms. UCRL-50564, Rev. 1., University of California, Lawrence Livermore National Laboratory, Livermore, California.

(TM83) Till, J.E., and H.R. Meyer, eds. 1983. Radiological Assessment. NUREG/CR-3332, ORNL-5968, U.S. Nuclear Regulatory Commission, Washington, D.C.

(USDA83) U.S. Department of Agriculture (USDA). 1983. Food Consumption: Households in the United States, Seasons and Years 1977-1978. National Food Consumption Survey 1977-1978, Report No. H-6, Human Nutrition Information Service, Consumer Nutrition Division. U.S. Government Printing Office, Washington, D.C.

(Va82) Van Bruwaene, R., G.B. Gerber, R. Kirchmann, and J. Colard. 1982. "Metabolism of antimony-124 in lactating dairy cows." Health Physics, 43:733-738.

(Va83) Van Den Hoek, J., M.H.J. Ten Have, and G.B. Gerber. 1983. "The metabolism of tritium and water in the lactating dairy cow." Health Physics, 44:127-133.

(Va84) Van Bruwaene, R., G.B. Gerber, R. Kirchmann, J. Colard, and J. Van Kerkom. 1984.

"Metabolism of ${ }^{51} \mathrm{Cr},{ }^{54} \mathrm{Mn},{ }^{59} \mathrm{Fe}$, and ${ }^{60} \mathrm{Co}$ in lactating dairy cows." Health Physics, 46:1069-1082.

(Vo93) Voight, G., H. Muller, H.G. Paretzke, T. Bauer, and G. Rohrmoser. 1993. "Cs-137 transfer after Chernobyl from fodder into chicken meat and eggs." Health Physics, 65:141-146. 


\section{Appendix A}

\section{List of the Elements}




\section{List of Elements}

\begin{tabular}{|c|c|c|c|c|c|c|c|c|}
\hline $\begin{array}{l}\text { Atomic } \\
\text { Number }\end{array}$ & Symbol & Name & $\begin{array}{c}\text { Atomic } \\
\text { Number }\end{array}$ & Symbol & Name & $\begin{array}{c}\text { Atomic } \\
\text { Number }\end{array}$ & Symbol & Name \\
\hline 1 & $\mathrm{H}$ & hydrogen & 38 & $\mathrm{Sr}$ & strontium & 75 & $\operatorname{Re}$ & rhenium \\
\hline 2 & $\mathrm{He}$ & helium & 39 & $\mathrm{Y}$ & yttrium & 76 & Os & osmium \\
\hline 3 & $\mathrm{Li}$ & lithium & 40 & $\mathrm{Zr}$ & zirconium & 77 & Ir & iridium \\
\hline 4 & $\mathrm{Be}$ & beryllium & 41 & $\mathrm{Nb}$ & niobium & 78 & $\mathrm{Pt}$ & platinum \\
\hline 5 & B & boron & 42 & Mom & nolybdenum & 79 & $\mathrm{Ai}$ & gold \\
\hline 6 & $\mathrm{C}$ & carbon & 43 & $\mathrm{Tc}$ & technetium & 80 & $\mathrm{Hg}$ & mercury \\
\hline 7 & $\mathrm{~N}$ & nitrogen & 44 & $\mathrm{Ru}$ & ruthenium & 81 & $\mathrm{Tl}$ & thallium \\
\hline 8 & $\mathrm{O}$ & oxygen & 45 & $\mathrm{Rh}$ & rhodium & 82 & $\mathrm{~Pb}$ & lead \\
\hline 9 & $\mathrm{~F}$ & fluorine & 46 & $\mathrm{Pd}$ & palladium & 83 & $\mathrm{Bi}$ & bismuth \\
\hline 10 & $\mathrm{Ne}$ & neon & 47 & $\mathrm{Ag}$ & silver & 84 & Po & polonium \\
\hline 11 & $\mathrm{Na}$ & sodium & 48 & $\mathrm{Cd}$ & cadmium & 85 & At & astatine \\
\hline 12 & $\mathrm{Mg} \quad \mathrm{n}$ & magnesium & 49 & In & indium & 86 & $\mathrm{Rn}$ & radon \\
\hline 13 & $\mathrm{Al}$ & aluminum & 50 & $\mathrm{Sn}$ & tin & 87 & $\mathrm{Fr}$ & francium \\
\hline 14 & $\mathrm{Si}$ & silicon & 51 & $\mathrm{Sb}$ & antimony & 88 & $\mathrm{Ra}$ & radium \\
\hline 15 & $\mathrm{P}$ & phosphorus & 52 & $\mathrm{Te}$ & tellurium & 89 & Ac & actinium \\
\hline 16 & $\mathrm{~S}$ & sulfur & 53 & I & iodine & 90 & Th & thorium \\
\hline 17 & $\mathrm{Cl}$ & chlorine & 54 & $\mathrm{Xe}$ & xenon & 91 & $\mathrm{~Pa}$ & protactinium \\
\hline 18 & $\mathrm{Ar}$ & argon & 55 & $\mathrm{Cs}$ & cesium & 92 & $\mathrm{U}$ & uranium \\
\hline 19 & $\mathrm{~K}$ & potassium & 56 & $\mathrm{Ba}$ & barium & 93 & $\mathrm{~Np}$ & neptunium \\
\hline 20 & $\mathrm{Ca}$ & calcium & 57 & $\mathrm{La}$ & lanthanum & 94 & $\mathrm{Pu}$ & plutonium \\
\hline 21 & $\mathrm{Sc}$ & scandium & 58 & $\mathrm{Ce}$ & cerium & 95 & $\mathrm{Am}$ & americium \\
\hline 22 & $\mathrm{Ti}$ & titanium & 59 & Prpra & aseodymium & 96 & $\mathrm{Cm}$ & curium \\
\hline 23 & $\mathrm{~V}$ & vanadium & 60 & $\mathrm{Nd} \mathrm{r}$ & neodymium & 97 & $\mathrm{Bk}$ & berkelium \\
\hline 24 & $\mathrm{Cr}$ & chromium & 61 & $\mathrm{Pm} \mathrm{p}$ & promethium & 98 & $\mathrm{Cf}$ & californium \\
\hline 25 & Mn & manganese & 62 & $\mathrm{Sm}$ & samarium & 99 & Es & einsteinium \\
\hline 26 & $\mathrm{Fe}$ & iron & 63 & $\mathrm{Eu}$ & europium & 100 & Fm & fermium \\
\hline 27 & $\mathrm{Co}$ & cobalt & 64 & $\mathrm{Gd}$ & gadolinium & 101 & $\mathrm{Md}$ & mendelevium \\
\hline 28 & $\mathrm{Ni}$ & nickel & 65 & $\mathrm{~Tb}$ & terbium & 102 & No & nobelium \\
\hline 29 & $\mathrm{Cu}$ & copper & 66 & Dy & dysprosium & 103 & $\mathrm{Lr}$ & lawrencium \\
\hline 30 & $\mathrm{Zn}$ & zinc & 67 & Ho & holmium & 104 & $\mathrm{Rf}$ & rutherfordium \\
\hline 31 & $\mathrm{Ga}$ & gallium & 68 & Er & erbium & 105 & $\mathrm{Ha}$ & hafnium \\
\hline 32 & $\mathrm{Ge}$ & germanium & 69 & $\mathrm{Tm}$ & thulium & 106 & $\mathrm{Sg}$ & seaborgium \\
\hline 33 & As & arsenic & 70 & $\mathrm{Yb}$ & ytterbium & 107 & Ns & nielsbohrium \\
\hline 34 & $\mathrm{Se}$ & selenium & 71 & $\mathrm{Lu}$ & lutetium & 108 & Hs & hassium \\
\hline 35 & $\mathrm{Br}$ & bromine & 72 & $\mathrm{Hf}$ & hafnium & 109 & Mt & meitnerium \\
\hline 36 & $\mathrm{Kr}$ & krypton & 73 & $\mathrm{Ta}$ & tantalum & & & \\
\hline 37 & $\mathrm{Rb}$ & rubidium & 74 & W & tungsten & & & \\
\hline
\end{tabular}




\section{Distribution}

No. of

Copies

\section{OFFSITE}

S. Harris

Confederated Tribes of the Umatilla

Indian Reservation

P.O. Box 638

Pendleton, OR 97801

T. A. Ikenberry

Dade Moeller \& Associates

1845 Terminal Drive, Suite 140

Richland, WA 99352-

J. M. Leitch

U.S. Environmental Protection Agency

Region 10

1200 Sixth Ave

Seattle, WA 98101

R. G. Schreckhise

Washington State University Tri-Cities

2710 University Drive

Richland, WA 99352-1671

4 Washington State Department of Health

Division of Radiation Protection

7171 Cleanwater Lane, Building 5

P.O. Box 47827

Olympia, WA 98504-7827

ATTN: A. W. Conklin

R. Evans

J. J. Martell

J. W. Schmidt
No. of

Copies

ONSITE

3 DOE Richland Operations Office
E. M. Bowers
A2-15
M. F. Jarvis
A5-15
D. C. Ward
A2-17

Duratek Federal Services, Inc .
A. V. Savino,
H1-11

2 Fluor Federal Services

P. D. Rittman

E6-17

J. C. Van Keuren

L6-57

3 Fluor Hanford

L. P. Diediker

$\mathrm{N} 1-24$

D. L. Klages

L5-66

D. J. Rokkan

N1-24

23 Pacific Northwest National Laboratory
E. J. Antonio
K3-54
G. R. Bilyard
K3-54
B. A. Napier
K3-54
T. T. Jarvis
K6-80
C. T. Kincaid
K9-33
T. M. Poston
K6-75
K. Rhoads
K3-54
S. F. Snyder
K3-54
D. L. Strenge (2)
K3-54
L. H. Staven (5)
K3-54
R. D. Stenner
$\mathrm{K} 3-54$
Information Release Office (7) K1-06

Distr.1 